\title{
Dual-Energy CT in Head and Neck Imaging
}

\author{
Elise D. Roele ${ }^{1}$ - Veronique C. M. L. Timmer ${ }^{2}$ - Lauretta A. A. Vaassen ${ }^{2}$. \\ Anna M. J. L. van Kroonenburgh ${ }^{1}$ A. A. Postma ${ }^{1}$
}

Published online: 29 March 2017

(c) The Author(s) 2017. This article is an open access publication

\begin{abstract}
Purpose of Review To explain the technique of Dual-energy CT (DECT) and highlight its applications and advantages in head and neck radiology.

Recent Findings Using DECT, additional datasets can be created next to conventional images. In head and neck radiology, three material decomposition algorithms can be used for improved lesion detection and delineation of the tumor. Iodine concentration measurements can aid in differentiating malignant from nonmalignant lymph nodes and benign posttreatment changes from tumor recurrence. Virtual non-calcium images can be used for detection of bone marrow edema. Virtual mono-energetic imaging can be useful for improved iodine conspicuity at lower $\mathrm{keV}$ and for reduction of metallic artifacts and increase in signal-tonoise ratio at higher $\mathrm{keV}$.
\end{abstract}

This article is part of the Topical Collection on ENT Imaging.

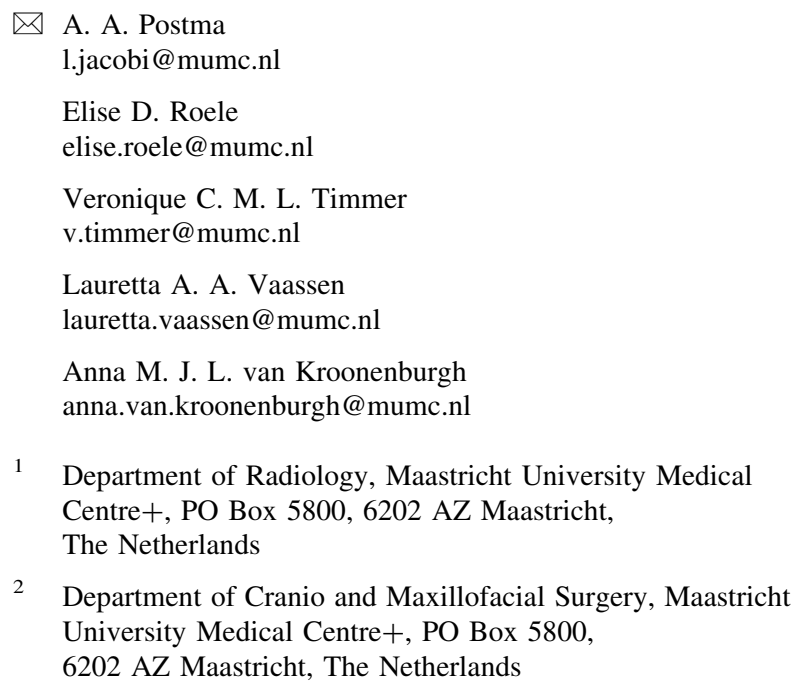

1 Department of Radiology, Maastricht University Medical Centre+, PO Box 5800, 6202 AZ Maastricht, The Netherlands

2 Department of Cranio and Maxillofacial Surgery, Maastricht University Medical Centre+, PO Box 5800, 6202 AZ Maastricht, The Netherlands

Summary DECT and its additional reconstructions can play an important role in head and neck cancer patients, from initial diagnosis and staging, to therapy planning, evaluation of treatment response and follow-up. Moreover, it can be helpful in imaging of infections and inflammation and parathyroid imaging as supplementary reconstructions can be obtained at lower or equal radiation dose compared with conventional single energy scanning.

Keywords Dual-energy CT $\cdot$ Spectral CT $\cdot$ Head and neck cancer · Lymph node imaging · Parathyroid adenoma . Metal artifact reduction

$\begin{array}{ll}\text { Abbreviations } \\ \text { ALARA } & \text { As low as reasonably achievable } \\ \text { BME } & \text { Bone marrow edema } \\ \text { CNR } & \text { Contrast-to-noise ratio } \\ \text { CT } & \text { Computed tomography } \\ \text { CTA } & \text { Computed tomography angiography } \\ \text { CE-CT } & \text { Contrast-enhanced CT } \\ \text { DECT } & \text { Dual-energy CT } \\ \text { HNSCC } & \text { Head and neck squamous cell carcinoma } \\ \text { HU } & \text { Hounsfield units } \\ \text { IMAR } & \text { Iterative metal artifact reduction } \\ \text { IOM } & \text { Iodine overlay map } \\ \text { keV } & \text { Kilo electron volt } \\ \text { kVp } & \text { Peak kilo electron volt } \\ \text { MDCT } & \text { Multidetector CT } \\ \text { MR(I) } & \text { Magnetic resonance imaging } \\ \text { OC image } & \text { Optimal contrast image } \\ \text { OPTkeV } & \text { Optimized image quality keV } \\ \text { PET } & \text { Positron emission tomography } \\ \text { PTA } & \text { Peritonsillar abscess } \\ \text { SECT } & \text { Single energy CT } \\ \text { SNR } & \text { Signal-to-noise ratio } \\ \end{array}$


SCC

Squamous cell carcinoma

VMI

Virtual mono-energetic imaging

$\mathrm{VNCa}$

Virtual non-calcium

$\mathrm{VNC}$

Virtual noncontrast

WA

Weighted average

\section{Introduction}

Imaging is a cornerstone of the diagnostic work-up of patients with suspected head and neck pathology, especially in the diagnosis and staging of head and neck cancer, but also in inflammatory processes, abscesses, and lymph node imaging. The available imaging arsenal expanded in the recent decades from conventional radiography, fluoroscopy, and ultrasound to more sophisticated imaging modalities like $\mathrm{CT}$ and MRI including advanced $\mathrm{CT}$ and MR techniques as well as hybrid imaging as are PET/CT and PET/MR.

Starting in the late eighties, CT shifted from sequential scanning to spiral or helical scanning. This was soon followed by the introduction of multidetector row imaging. At the moment CT scanners with detectors up to 320 rows are clinically available. These developments increased the imaging quality as regard to temporal and spatial resolution. The ongoing development in the computational abilities, e.g. in iterative reconstructions, increases the quality and clinical applications of CT even further.

It has only been since the last decade that Dual-energy CT (DECT) became clinically available. By means of two X-ray spectra instead of one, DECT offers increased capabilities and advantages over single energy scanning methods, such as the potential for material characterization and differentiation and the calculation of virtual mono-energetic reconstructions. In the early years applications of CT were mainly in thoracic, abdominal and vascular imaging fields, for example, automated bone removal in CTA, quantification of the amount of iodine in renal masses, and detection of perfusion defects in CTA in patients with pulmonary embolism [1-6]. Later, this was followed by a more widespread implementation in other areas of the body as in the imaging of gout $[7,8]$. Initially brain and head and neck applications fell somewhat behind, both now, however, trying to catch up with others [9-11].

In this article, we will explain the basics of the technique of Dual-energy CT, and its applications and advantages in head and neck radiology.

\section{DECT Technique}

The density of the tissues in CT is calculated by the attenuation coefficient expressed in Hounsfield units (HU). The difference between the numbers of the photons emitted and that of the detected ones equals the attenuation and is determined by the interaction of photons with and within the tissue. In radiology, these interactions are mainly determined by photon absorption (photoelectric effect) and scattering (Compton effect). The variation in Compton effects at the energy levels used in CT is relatively small across different materials, while the photoelectric effect varies considerably and is strongly dependent on the atomic number $(Z)$ of the material and the photon energy $\left((Z / E)^{3}\right)$ (Fig. 1).

The photoelectric effect increases with the increasing atomic number. Commonly used contrast media like iodine $(Z=53)$ and barium $(Z=56)$ have strong photoelectric effects resulting in a high attenuation, especially at the lower energies, because of reaching the k-edge. Most of the tissues in the human body consist of lower effective atomic numbers, such as fat and water, which show relatively weak photoelectric effect and attenuation. In these tissues, the Compton effect prevails. An exception is calcium $(Z=20)$ which has a relative high atomic number compared with the other (soft) tissues of the human body and therefore shows in comparison a higher photoelectric effect and attenuation (Fig. 2).

The attenuation also depends on the photon energy $(\mathrm{KeV})$ and is determined by the maximum voltage level of the X-ray tube $(\mathrm{kVp})$. In Fig. 2, we can visualize that when scanning with two different energies, there is a difference in the attenuation coefficients of the tissues. This difference in attenuation at different energies rests at the basis of DECT scanning.

In single energy CT (SECT) scanners, one single polychromatic energy spectrum is used for imaging, whereas in DECT, two X-ray spectra are needed. In the initial description of DECT by Hounsfield, objects were scanned twice. Today, there are multiple ways to perform DECT scanning [12, 13]. Different DECT techniques include systems with a double X-ray tube, systems with a single tube which can change the $\mathrm{kVp}$ setting, with a filter or a dual-layer detector (Fig. 3).

The evaluation of the acquired data by DECT scanning gets reconstructed into low- and high-energy datasets, in which 80 and $140 \mathrm{kVp}$ constitute the frequently encountered combination. The two datasets can be combined to a single mixed-image dataset, also called linear blending or weighted average, resembling a SECT. With a ratio of 0.3 (30\% $80 \mathrm{kVp}$ and $70 \% 140 \mathrm{kVp}$ ) a conventional, single energy data image set at $120-\mathrm{kVp}$ acquisition is simulated $[3,14]$. By shifting the percentage of contribution of each dataset (linear blending), one can either choose to move to higher-energy contribution for the increased signal-to-noise ratio (SNR) and artifact reduction or a lower-energy contribution for the improved contrast-to-noise ratio (CNR) and lesion conspicuity [12]. Next to linear blending, 

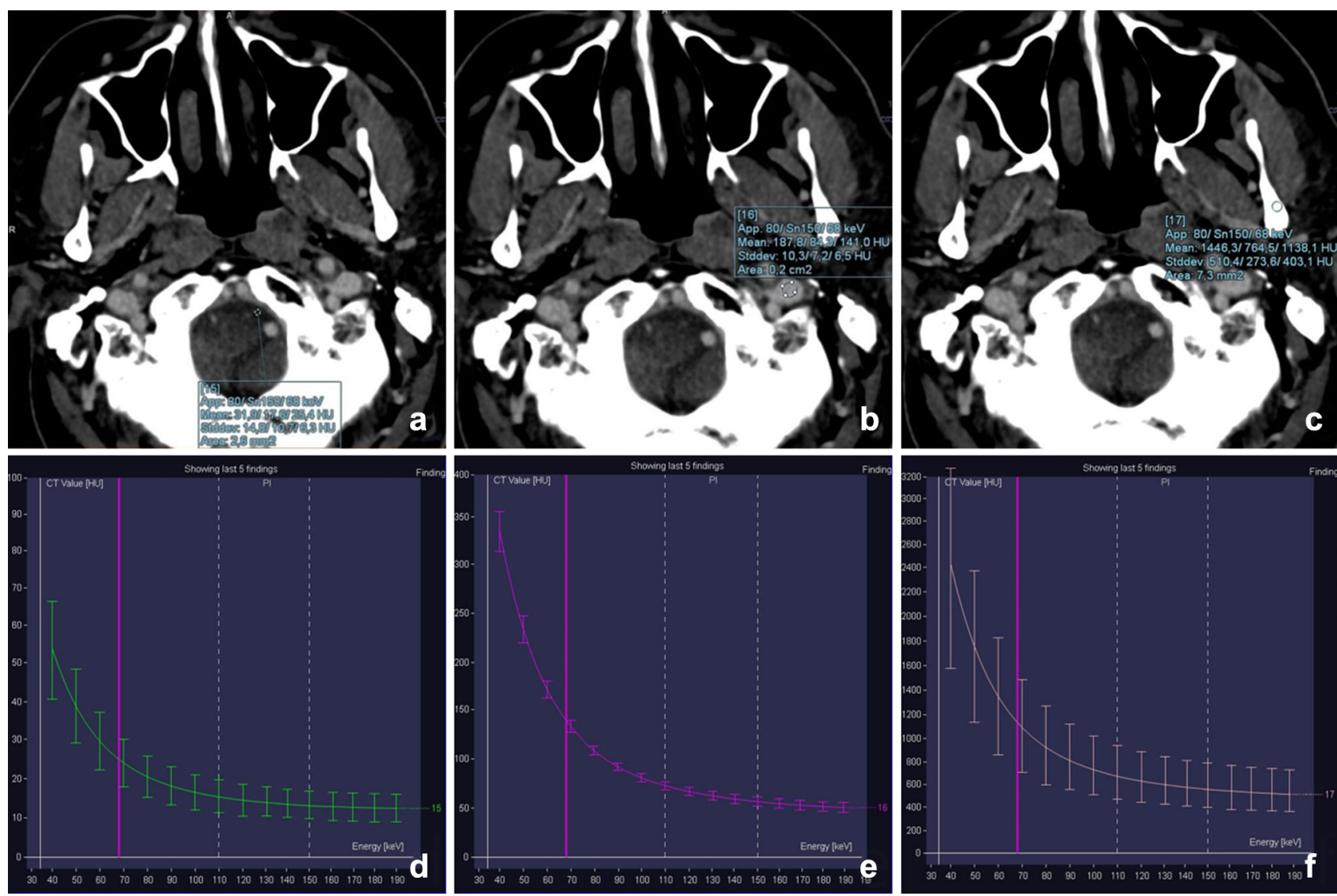

Fig. 1 The spectral curves depicted from a DECT of the neck are shown for cerebrospinal fluid (water $\mathbf{a}, \mathbf{d}$ ), vessels (iodine $\mathbf{b}, \mathbf{e}$ ), and bone (calcium c, f). Hounsfield units (HU) are plotted against the

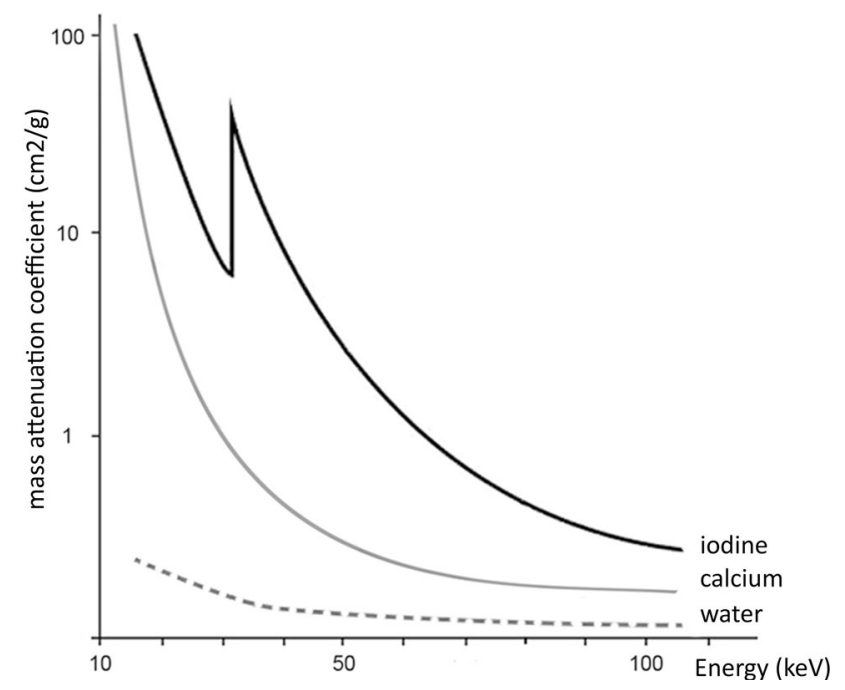

Fig. 2 Attenuation curves of iodine, calcium, and water plotted against energy $(\mathrm{keV})$. At lower energy, the attenuation of iodine $(Z=53)$ is increasing with an additional increase at the k-edge. The attenuations of calcium $(Z=20)$ and water are significantly lower than that for iodine, providing the possibility for material differentiation in DECT mono-energetic energies ranging from 30 to $190 \mathrm{keV}$. Note that the HU scale differs for each plot. At lower energy, the HU increases, especially in calcium and iodine

nonlinear blending functions have been developed to optimize the blending processing [15]. Datasets are mixed via a computational function e.g. a sigmoidal function to maximize contrast and lower noise, which should provide an optimal contrast image [16].

Virtual monochromatic imaging (VMI) allows for image reconstruction at different virtual monochromatic energies instead of using a polychromatic spectrum. At higher virtual energies, beam hardening artifacts can be reduced and SNR is increased. At lower virtual monochromatic energies there is increased conspicuity of iodine (at the cost of lower SNR). These VMI images are reconstructed for a specific purpose and are commonly used in addition to the standard (blended) reconstructions [17-19]. By use of VMI at different energies, a spectral attenuation curve as a function of energy can be plotted [19] (Fig. 1). More recently, advanced algorithms for VMI are allowing an increased SNR at lower virtual energies by using an advanced calculation with the use of a split frequency filter [20, 21].

One of the major strengths of DECT is material differentiation and characterization. Material specific images for identifying or differentiating certain materials/tissues 

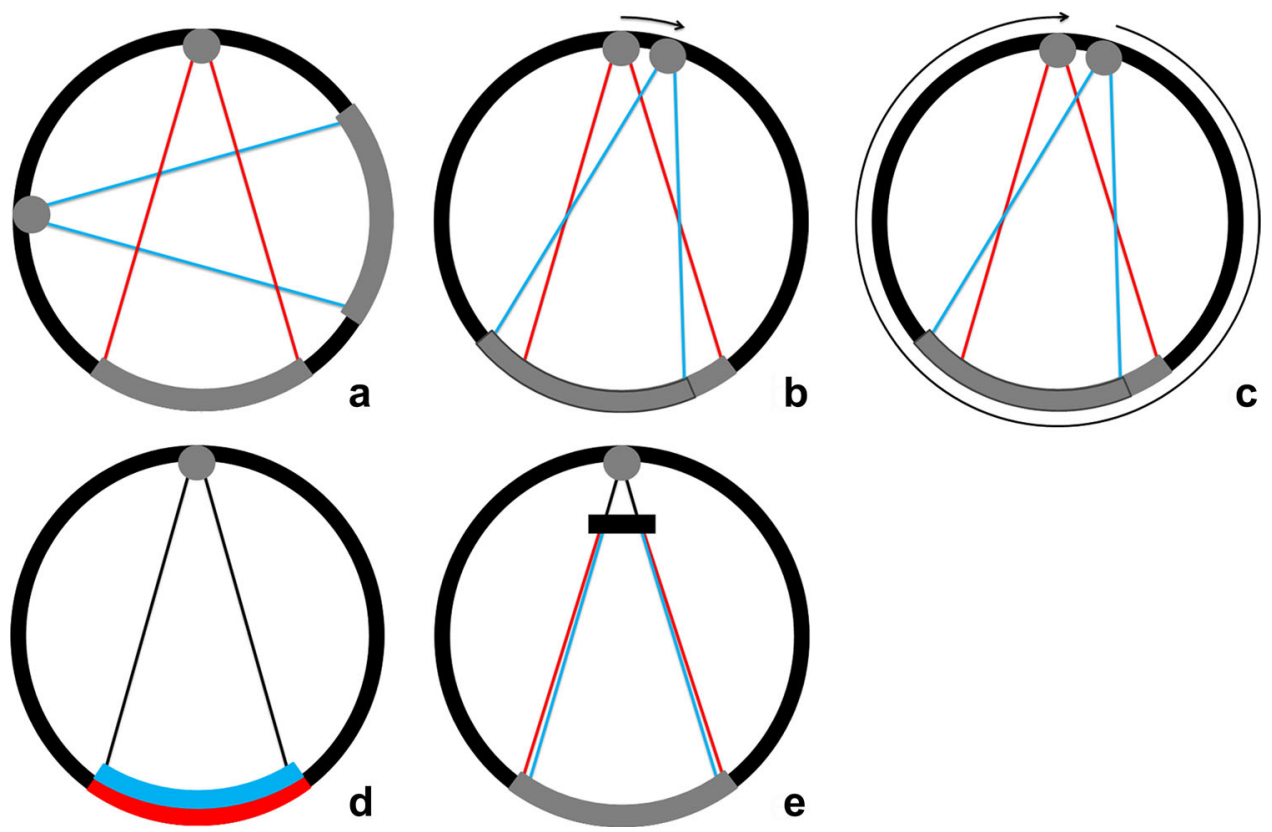

Fig. 3 DECT systems. Dual-source dual-energy system (a): two separate X-ray tubes and detectors are orthogonally mounted for simultaneous data acquisition and processing. Each tube can be set at different voltage levels. The $\mathrm{kVp}$ settings can be adjusted between 70 and $\mathrm{Sn} 150 \mathrm{kVp}$ for the latest generation dual-source scanner (Siemens). Single-source dual-energy system: switching the $\mathrm{kVp}$ setting can generate two spectra. One method is fast $\mathrm{kV}$ switching (b), in which one X-ray tube rapidly switches between low and high $\mathrm{kVps}$ (GE). The second method is $\mathrm{kVp}$ switching between single rotations (c), the so-called dual spiral approach (Toshiba, Siemens). A single

are calculated by using the unique linear attenuation coefficient of the specific materials (fat, calcium, iodine and water). In a 3-material algorithm, the concentration of e.g. iodine or calcium can be calculated. When the iodine concentration is known, it can be subtracted from the mixed dataset to generate a virtual noncontrast (VNC) image. The iodine map can be superimposed in color on the gray-scale image to create fusion images. Similar techniques are used to generate virtual non calcium (VNCa) images.

Next to VMI and material differentiation, the effective $\mathrm{Z}$ number and electron density can be calculated from the datasets.

Initially, there have been concerns about higher radiation dose of DECT compared to conventional CT scanners, reported up to three times as high [22]. With advances in technology the radiation dose has lowered and is now comparable to, or even less than, conventional SECT scanners. Either by direct lowering of the dose or the potential of replacing the nonenhanced scan by VNC images and therefore indirect dose reduction [10, 23, 24]. This opens up the wider use of DECT in clinical applications. detector then processes the information from both voltage levels. Dual-layer system (d): a polychromatic spectrum from one tube passes on to a dual-layer detector. The upper layer is sensitive to the low-energy photons, while the second layer processes the high-energy photons. The combination of both detectors creates the combined image (Phillips). Single-source twin-beam system (e): the single $\mathrm{X}$-ray beam is pre-filtered between the tube and the patient by gold $\mathrm{Au}$ ) and tin (Sn) filter. The $120-\mathrm{kVp} \mathrm{X}$-ray beam is split into high( $\mathrm{Sn})$ and low-energy $(\mathrm{Au})$ spectra (Siemens)

\section{Applications}

\section{Metal Artifact Reduction}

Metallic hardware can negatively affect image quality of surrounding tissues because of artifacts. This limitation is especially relevant in imaging of the oral cavity, with the frequent appearance of dental restorations, prosthetics, braces and metallic implants.

Metal artifacts on CT imaging are mainly caused by photon starvation or beam hardening effects by the high attenuation of metal. Photon starvation occurs when an $\mathrm{X}$-ray beam is completely absorbed by an object and an insufficient number of photons reach the detector to reconstruct an image (zero admission). Beam hardening artifacts are caused by absorption of the low energy photons of the polychromatic X-ray beam. The detected X-ray beam is 'hardened', containing higher average energy photons than expected. This results in streaks or dark-like bands around the object [25]. Since the clinical introduction of DECT, this technique has proven to be beneficial in the metal artifact reduction arsenal. Especially reconstructed monochromatic imaging is effective in reducing 
beam hardening artifacts by the absence of spectral shifts, which are present in polychromatic imaging [26] (Figure 4). VMI reconstructions of high energy levels can reduce metal artifacts and increase image quality [27-34] (Figs. 5, 6). Stolzmann et al. described the use of DECT versus SECT for metal artifact reduction in dental restorations. They found that the use of increasing energy VMI significantly reduced the amount of beam hardening artifacts caused by dental restorations. Image artifacts were lower on VMI than on conventional SECT [34]. Tanaka et al. evaluated different VMIs at $100,190 \mathrm{keV}$ and fused DECT images, resembling conventional $120-\mathrm{keV}$ CT imaging in living patients with dental implants. They concluded that $100 \mathrm{keV}$ VMI was superior to $190-\mathrm{keV}$ VMI and fused DECT imaging in reducing dark band-like metal artifacts caused by dental implants and additionally resulted in better adjacent bone diagnosis around the implants [27]. In a human cadaver study, De Crop et al. compared metal artifact reduction methods to conventional SECT of the oral cavity. High energy VMI $(140 \mathrm{keV})$ of DECT not only resulted in significant artifact reduction and better image quality but also reduced the low contrast resolution [35]. However, in their study, model based iterative reconstructions (IMAR) seemed to be the most promising metal artifact reduction technique for increasing image quality without adversely affecting contrast resolution [35]. Bongers et al. compared DECT based and iterative metal artifact reduction on hip prosthesis and dental hardware. Although IMAR showed a significantly higher reduction of metal artifacts, compared to VMI of $130-\mathrm{keV}$ DECT images, the combination of DECT and IMAR resulted in a highly significant reduction of metal artifacts compared with IMAR alone [36].

The use of DECT in metal artifact reduction of cervical spinal implants has been evaluated by Guggenberger et al. and Zhou et al. [30, 32]. Both authors concluded that, compared to average weighted $120-\mathrm{kVp}$ image, higher-keV VMI improved image quality and reduced metal artifacts in patients with metal orthopaedic implants. Zhou et al. found the optimal VMI at $130 \mathrm{keV}$. Furthermore, Guggenberger et al. calculated the individually adjusted mono-energy for optimized image quality (OPTkeV) for different spinal levels and vendors of the spinal implants, which showed a range between 123 and $141 \mathrm{keV}$ [32].

The optimal energy levels for metal artifact reduction are generally found between 100 and $140 \mathrm{keV}$ [28-32, 35-37] (Fig. 6). However, the extent of artifact reduction also depends on location, geometry and material composition of the implant.

\section{Head and Neck Oncology}

\section{Primary Tumor Delineation}

Currently CT and MRI are the standard image modalities used for primary staging of head and neck squamous cell carcinoma (HNSCC). Accurate diagnostic staging is essential for proper treatment of HNSCC and patient survival. Important factors affecting initial treatment of HNSCC include primary site, size, location, proximity to bone or cartilage, status of cervical lymph nodes, previous treatment, and histology [38]. Both CT and MRI report
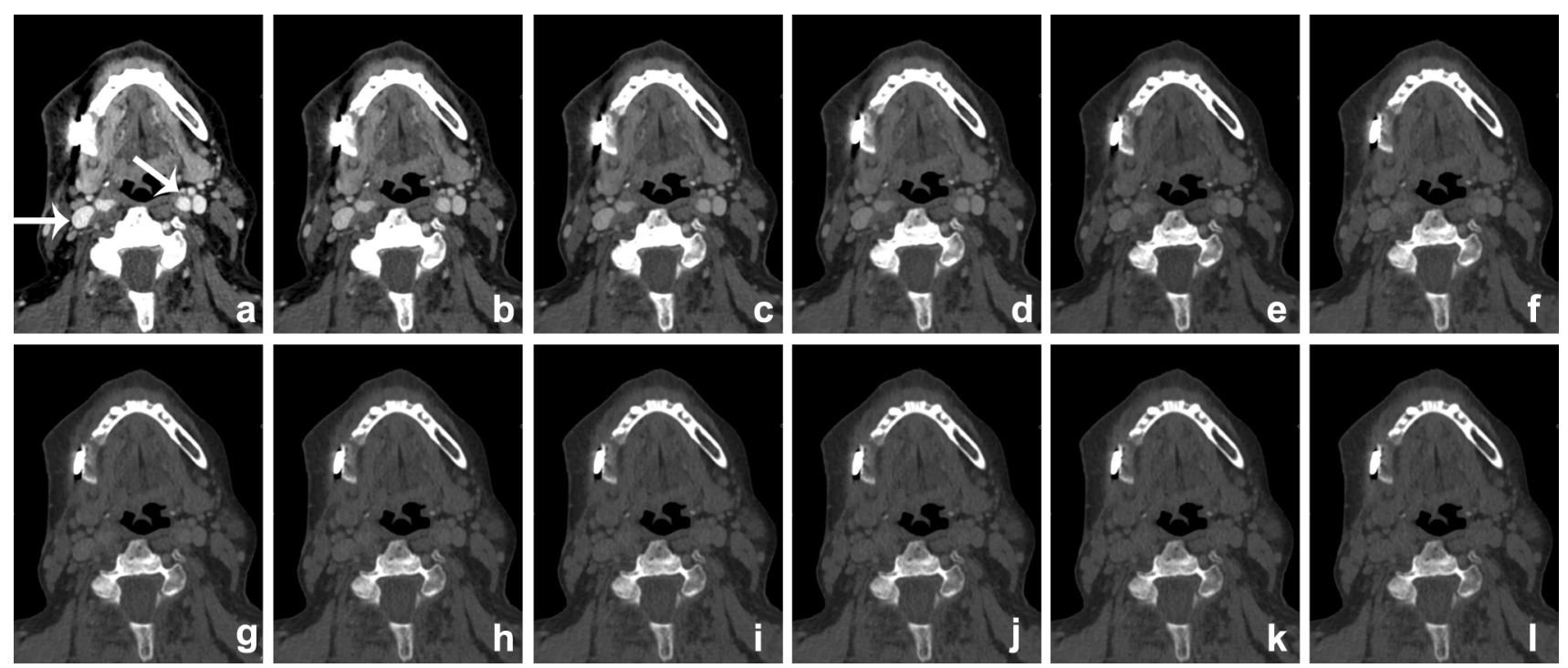

Fig. 4 VMI at the range of $40-150 \mathrm{keV}(\mathbf{a}-\mathbf{l}, 10-\mathrm{keV}$ interval) scanned after administration of iodinated contrast. The patient presented with an osteomyelitis of the mandible and cutaneous fistula (see also Fig. 12). Iodine conspicuity was increased at lower keV, as

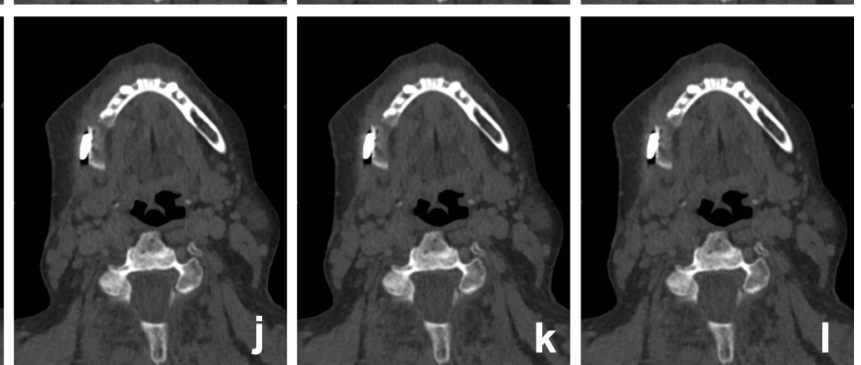

can be noticed by the increased CNR around the vessels (arrow). Higher-energy VMI results in an increase in signal-to-noise (SNR) and a decrease in streak artifacts caused by metallic hardware 

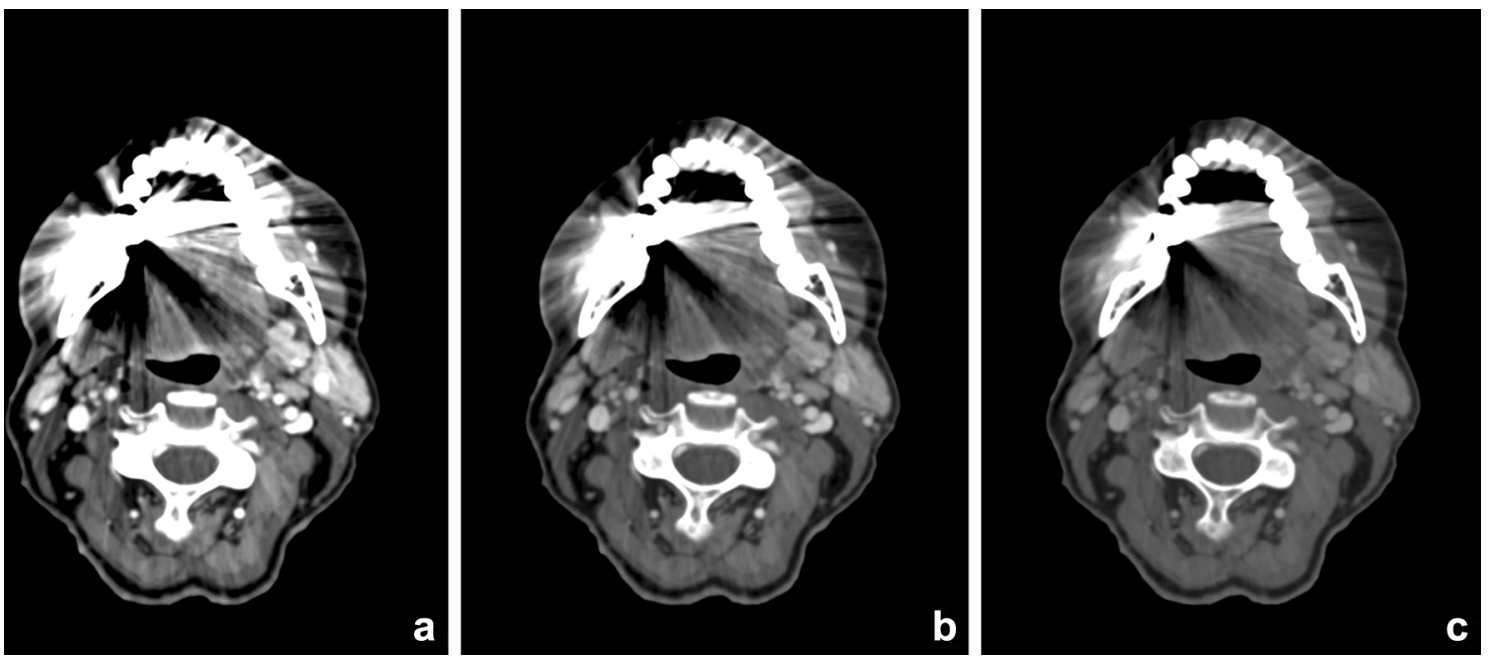

Fig. 5 Contrast-enhanced DECT of the oral cavity with VMIs at $55 \mathrm{keV}$ (a), $70 \mathrm{keV}(\mathbf{b})$, and $100 \mathrm{keV}$ (c). Significant beam-hardening artifacts in the oral cavity are present, due to dental fillings. These

artifacts are reduced at higher VMI. This is accompanied by an increase of SNR, whereas iodine conspicuity decreases
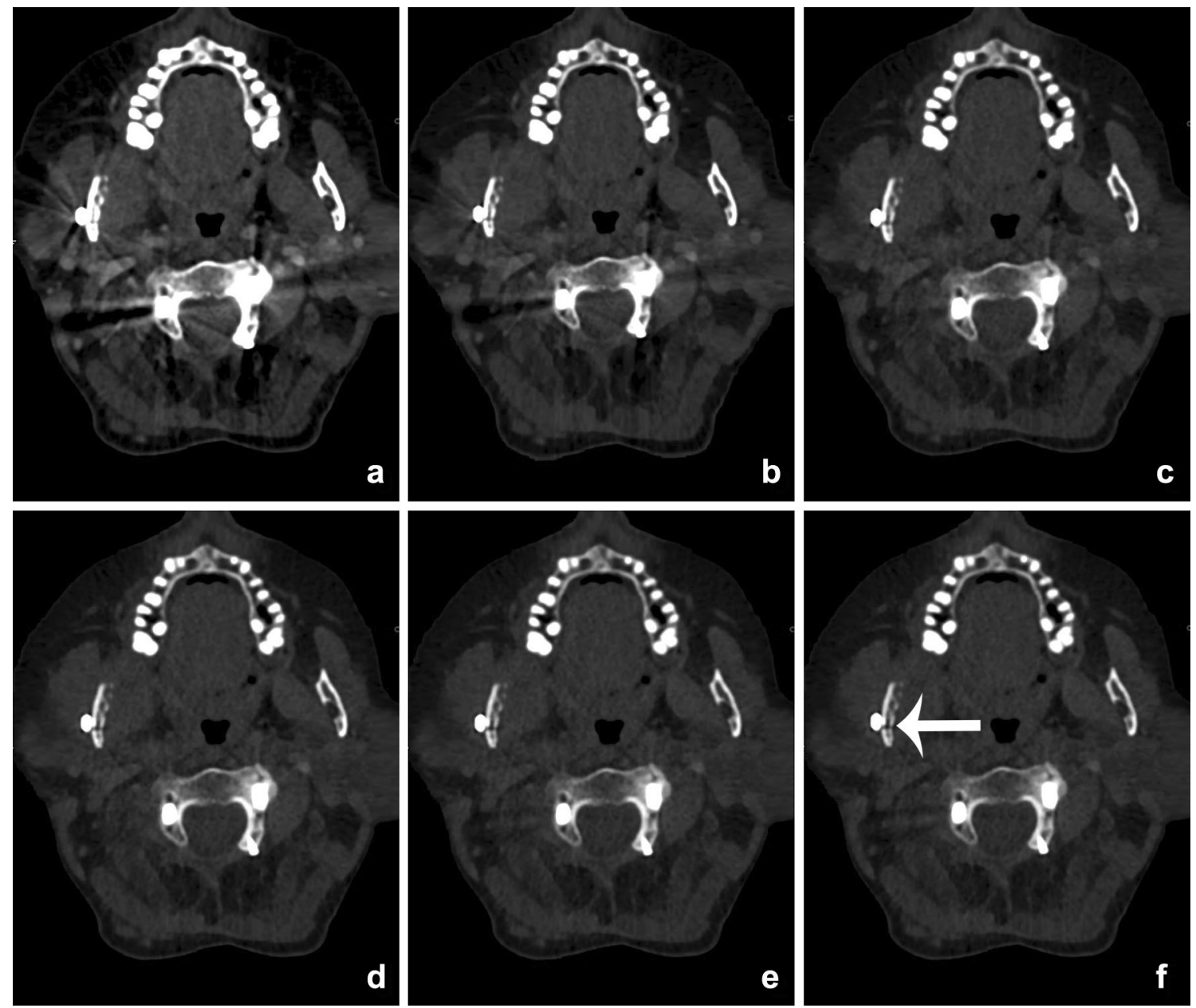

Fig. 6 VMIs at 60 (a), 80 (b), 100 (c), 120 (d), 140 (e), and $160 \mathrm{keV}$ (f) of a patient with a cervical spondylodesis and metallic hardware of the mandible (see also Fig. 12). With the increasing virtual mono-energetic energy, further reduction of metallic artifacts of both the cervical spondylodesis and the metallic hardware of the mandible can be seen. At lower $\mathrm{keV}$, streak artifacts obscure pathology at the right mandibular ramus. At higher keV, a fracture becomes visible at the mandible, which was undetectable at the mixed-imaging and low-keV images (arrow) 

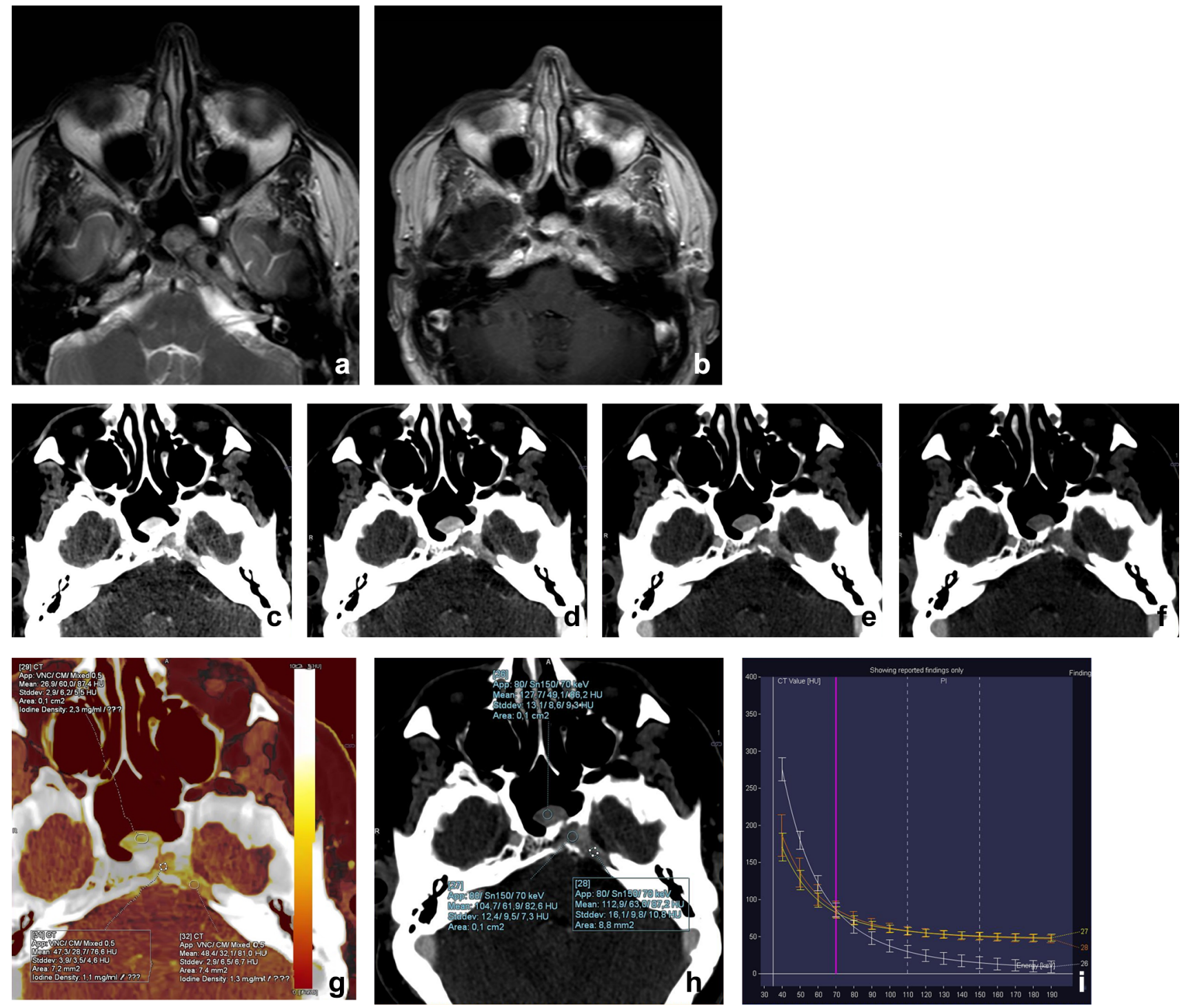

Fig. 7 A 48-year-old male presented with a traumatic skull base lesion. Initial CT demonstrated a lesion at the petroclival fissure, apex, and sphenoid sinus. MR with T2-weighted (a) and T1-weighted post-gadolinium images (b) showed the presence of the lesion with relatively low T2-signal and enhancement after gadolinium. By performing a transnasal biopsy of the sphenoid part, it would be feasible to obtain a histopathological diagnosis of the skull base lesion. On CT, the lesion is well appreciated with osteolysis of the petrous apex and enhanced after iodinated contrast. However, after

acceptable sensitivity and specificity for HNSCC staging in literature $[39,40]$. The evaluation of the tumor and infiltration of surrounded anatomical structures can be challenging. DECT, with its advantages in material characterization and differentiation, can be beneficial in primary HNSCC imaging. With the presence of two datasets at different $\mathrm{kVp}$ settings a variety of reconstructions can be made (Fig. 7).

Tawfik et al. demonstrated that images with a weighted average (WA) ratio of 0.6 and 0.8 in which the percentage of the lower energy is relatively high, the delineation of mono-energetic reconstructions $(\mathbf{c}-\mathbf{f}, 40-80 \mathrm{keV})$ and iodine fusion imaging, the lesion consists of two parts, with the medial part being more enhancing at lower $\mathrm{keV}$, with higher iodine uptake at fusion imaging $(\mathbf{g}, \mathbf{h})$. The graphs of the spectral curves (i) demonstrate two different attenuation curves. It was concluded that the medial part of the lesion was different from the lateral part and probably herniated pituitary after trauma. The more lateral lesion of the skull base still has no definitive diagnosis, because of the difficulty in accessing for biopsy. Thus far, no growth of the lesion is noted during follow-ups

head and neck tumors significantly improved compared to the WA 0.3 images which resemble a SECT of $120 \mathrm{kVp}$. Also the subjective image quality of the WA 0.6 images was superior to the WA 0.3 images [41]. In a more recent study, Scholtz et al. found the same trend of better tumor enhancement with increasing WA of $0.3,0.6$ and 0.8 in linear-blended imaging. In addition, Scholtz et al. compared a nonlinear image blending ("optimum contrast" application) to the different linearly blended images. In nonlinear-blended images the advantages of lower SNR at 

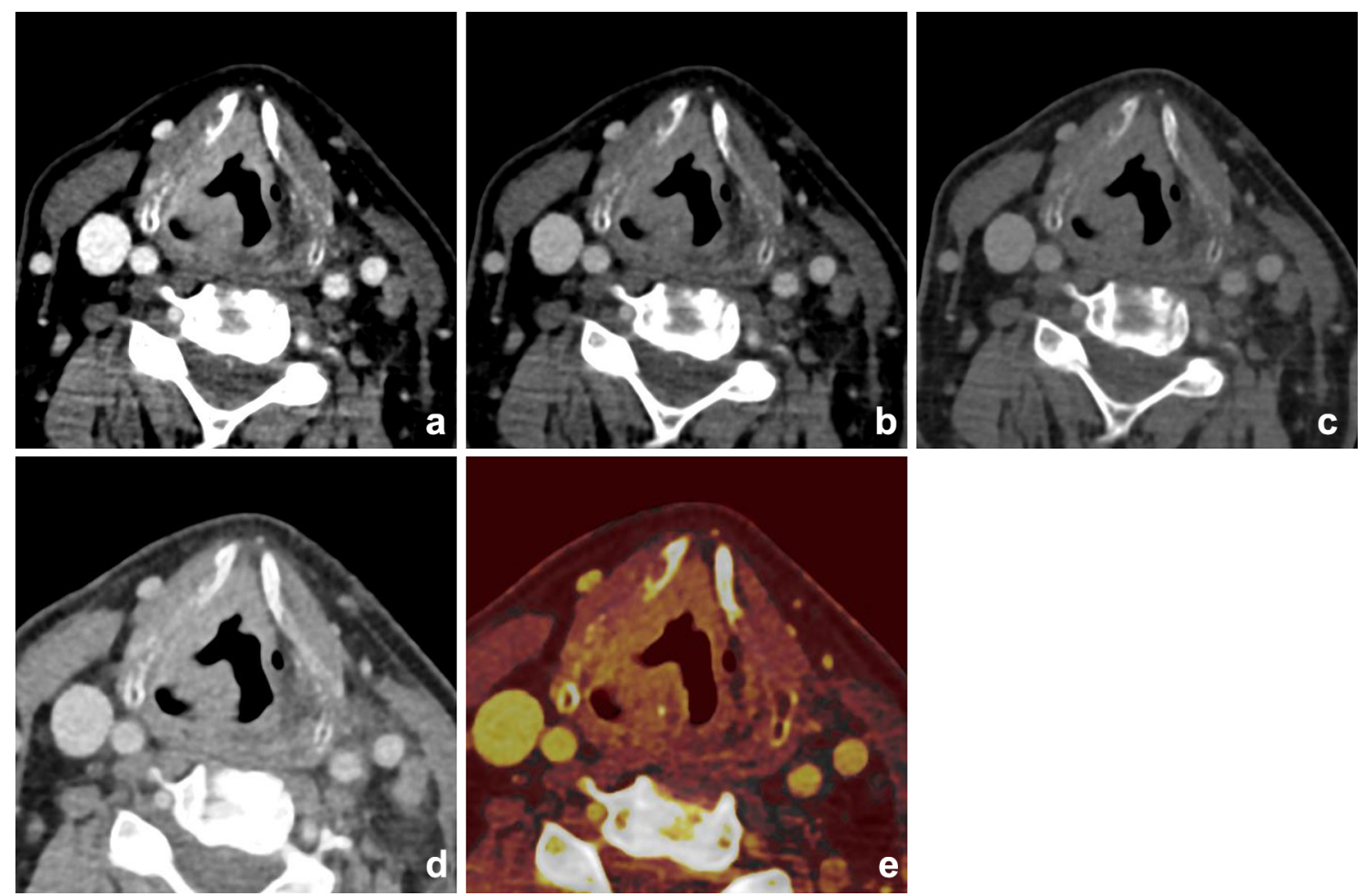

Fig. 8 Supralaryngeal carcinoma of the right hemilarynx in a 74-year-old male. Increased conspicuity of the tumor was shown at the lower virtual mono-energetic reconstructions (40 (a), 55 (b), and 70 (c) $\mathrm{keV}$ ). Note the increased differences between tumor and strap muscles at lower-keV settings, compared with mixed imaging (d) and higher-keV settings. These differences were even more enhanced on iodine fusion imaging (e). Also the extra-laryngeal extension was more easily appreciated at the iodine fusion images higher energies and the increased CNR in the lower energy spectrum are combined. This resulted in increased lesion conspicuity, while maintaining a good SNR [15, 42]. Furthermore, subjective overall image quality favored nonlinear-blended images [16].

In another study of Scholtz et al., blended $120-\mathrm{kVp}$ images were compared with $80-\mathrm{kVp}$ acquisition images. The mean tumor enhancement was higher at the $80-\mathrm{kVp}$ acquisition. Both were rated as good subjective image quality, but blended $120-\mathrm{kVp}$ images were rated superior to those of $80 \mathrm{kVp}$. The presence of image noise was increased at the $80-\mathrm{kVp}$ images, with a higher presence of metallic artifacts [43].

In a prospective study of Toepker et al. the identification of tumor margins in patients with oral cancer was compared in 80-, 140-kVp, mixed, and 'optimum contrast' (OC) DECT images. Low-kVp, mixed, and OC images all received good-to-excellent scores in image quality, while $140-\mathrm{kVp}$ images were rated as moderate to low. Contrast at the tumor margins was the highest for $80-\mathrm{kVp}$, mixed, and OC images compared with $140-\mathrm{kVp}$ images but the lowenergy images showed the highest image noise and were more prone to metal artifacts than the mixed and OC images. SNR was more favorable in mixed images [44].
VMI can also be applied for better tumor delineation in head and neck oncology [18] [45••]. Because of higher attenuation of iodine in lower-keV VMI, superior tumor contrast by iodine uptake can be achieved; this, however, can occur at the cost of higher image noise (Figs. 8, 9). Wichmann et al. investigated the value and image quality of VMI at different energy levels in patients with HNSCC. Objective enhancement of SCC lesion peaked in $40-\mathrm{keV}$, followed by $60-\mathrm{keV}$ reconstructions, but $60-\mathrm{keV}$ VMIs were subjectively more favorable in image quality and tumor delineation. Compared with linearly blended images, with a WA 0.3 , VMI of $60 \mathrm{keV}$ seemed to be superior in tumor attenuation and CNR [18].

Albrecht et al. evaluated the advanced application of VMI, (Mono+) and compared the images to linear-blended WA 0.3 DECT images in patients with head and neck cancer. The highest tumor attenuation was found at $40 \mathrm{keV}$ with a superior CNR compared with the WA 0.3 images. However, subjectively VMI at $55 \mathrm{keV}$ was preferred regarding image quality and tumor delineation [21]. Lam et al. evaluated VMI reconstructions of the head and neck with a single source fast-kV switching DECT. They found the highest SNR at 65-keV VMI for all tissues in head and neck imaging, but better tumor delineation and CNR at 

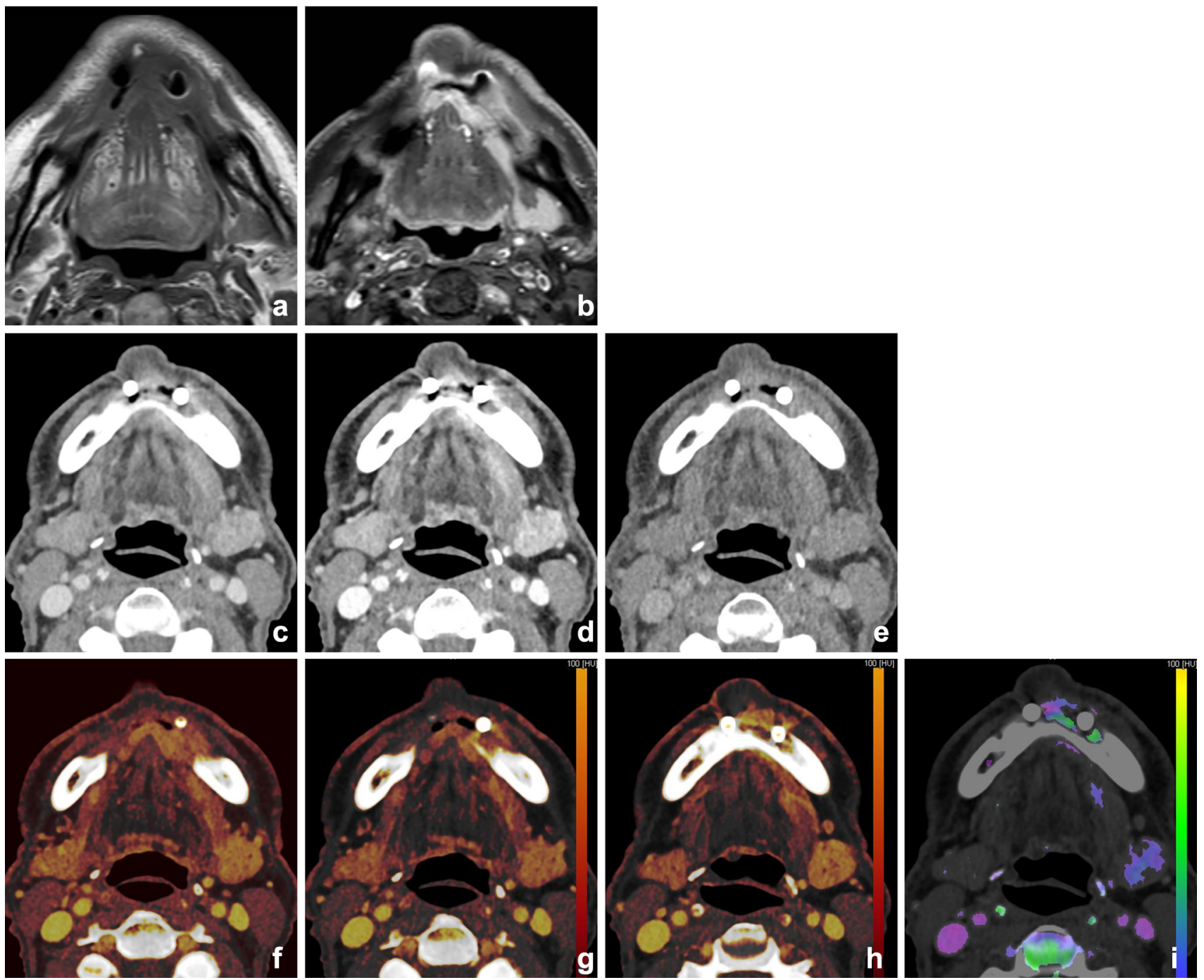

Fig. 9 A male with previous laryngeal cancer and oral implants presented with suspicion of oral cavity SCC at the site of the implants. MR showed considerable distortion and interpretation difficulties at the tumor site (T1-weighted (T1-w) (a). Fat-supressed T1-w after gadolinium (b) demonstrated abnormal signal and enhancement; however, delineation was challenging due to artifacts. $5 \mathrm{~mm}$ DECT

40-keV VMI [45••]. Based on their findings, Lam et al. recommend the use of a multiparametric approach with 65-keV VMI for general assessment of the neck, supplemented by $40-\mathrm{keV}$ VMI for better tumor differentiation to the surrounding soft tissue of the head and neck. Optimal SNR can thus vary depending on the scanner type and postprocessing techniques applied.

\section{Bone Invasion}

\section{Invasion of Thyroid Cartilage}

Accurate detection of cartilage invasion is of great importance for the appropriate treatment choice of

with soft kernel at 50\% linear blending (c), 90\% (d), and $10 \%$ (e) blending demonstrated the implants and osseous destruction of the mandible, better visualized at higher energies. Tumor enhancement was, however, difficult at mixed imaging, but was easily visualized at iodine fusion imaging $(\mathbf{f}-\mathbf{h})$. Bone marrow edema is demonstrated at BME imaging (i)

hypopharyngeal and laryngeal squamous cell carcinoma (SCC). Tumors without or with limited cartilage invasion can be treated with organ-preserved interventions including $\mathrm{CO}_{2}$-laser, minimally invasive surgery or (chemo)radiotherapy. Tumors with evident cartilage invasion require more aggressive treatment, frequently resulting in total laryngectomy, which may significantly impair the patient's quality of life [46].

Both CT and MRI imaging are currently used for the evaluation of cartilage invasion, each having its advantages and limitations. At CT, sensitivity and specificity of detection of cartilage invasion depend on the various diagnostic criteria of sclerosis, erosion, lysis, and extralaryngeal spread [47, 48]. Becker et al. reported that 

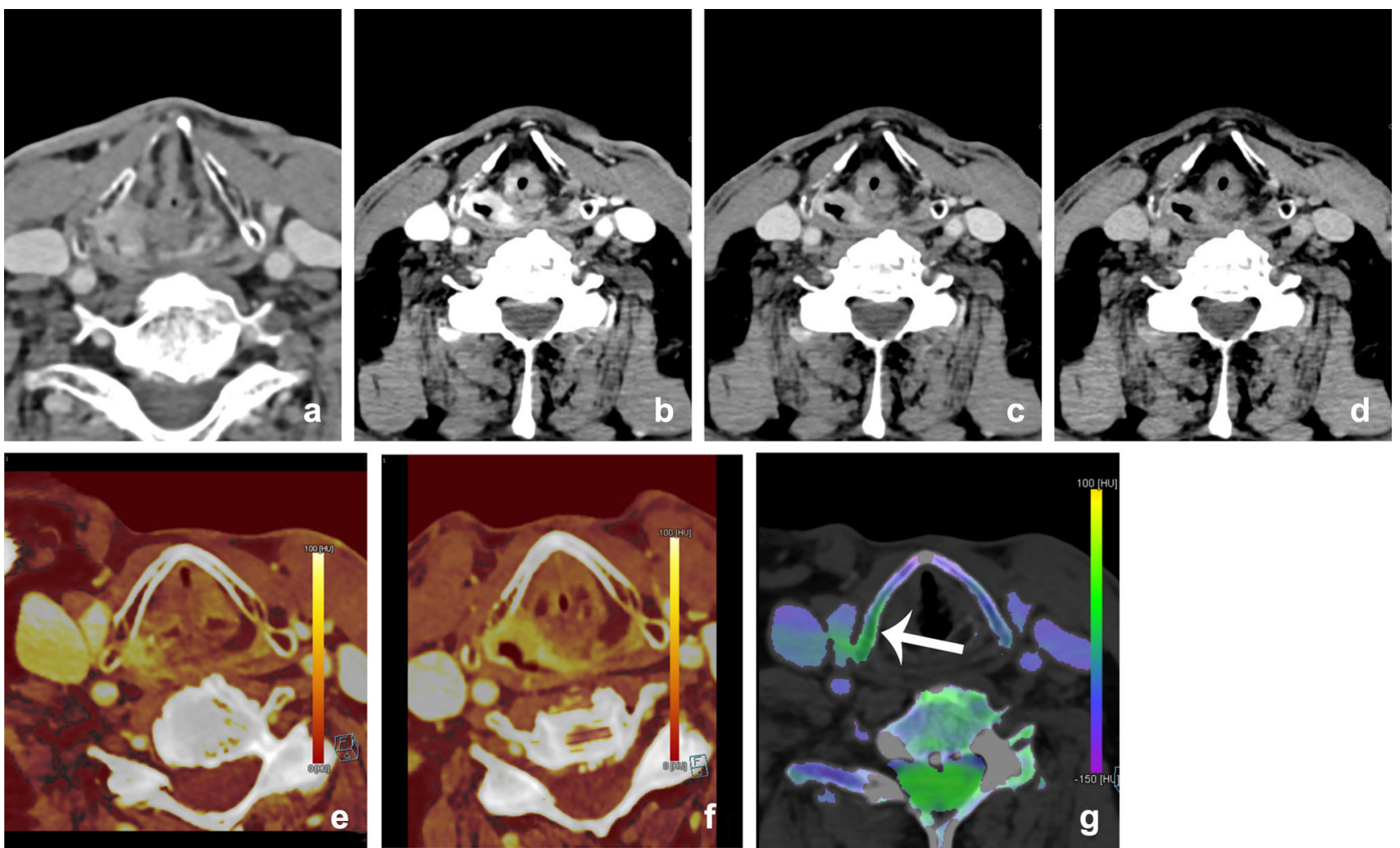

Fig. 10 Different reconstructions from one dataset. DECT of a 77-year-old male showed a right-sided piriform sinus SCC (a, mixed), suggestive of thyroid invasion. The tumor delineation is better depicted at the lower-energy images of the VMI (b: $40 \mathrm{keV}$; $\mathbf{c}$ : $70 \mathrm{keV}$; and $\mathbf{d}: 100 \mathrm{keV})$, and on the iodine fusion images $(\mathbf{e}, \mathbf{f})$. Bone marrow edema (BME) image (d), which demonstrates edema at the

sclerosis was the most sensitive criteria in all cartilages, but it could be due to reactive inflammation. An optimal combination of criteria yielded an overall sensitivity of $91 \%$, with a specificity of $68 \%[47,48]$. One of the problems with the use of SECT-imaging is the resemblance of attenuation of HNSCC with the attenuation of non-ossified laryngeal cartilage, making it difficult to accurately distinguish subtle cartilage invasion.

In a few recent studies, DECT has been shown to be beneficial in the evaluation of cartilage invasion by laryngeal and hypopharyngeal squamous cell carcinoma (SCC). Kuno et al. evaluated the combination of iodine overlay maps (IOM) and 0.3 WA images to evaluate cartilage invasion in hypopharyngeal and laryngeal SCC. IOM combined with WA imaging significantly improved the specificity of detection of laryngeal cartilage invasion compared with WA imaging alone with, respectively, 96 versus $70 \%$, while sensitivity remained at $86 \%$. Furthermore, the inter-observer reproducibility of evaluating cartilage invasion also improved [49].

The use of VMI in the assessment of cartilage invasion has been recently evaluated by a retrospective study of thyroid cartilage. Visualization of direct tumor invasion is the easiest way to demonstrate cartilage invasion; this can be demonstrated made more easily by means of lower-energy images from VMI and iodine fusion images. Demonstration of edema of the thyroid in virtual noncalcium images can serve as an additional argument for invasion of the thyroid (arrow)

Forghani et al. Thirty patients with laryngeal or hypopharyngeal SCC and 10 healthy patients underwent fast-kV switching DECT. It was shown that tumorous cartilage has a significantly different spectral $\mathrm{HU}$ curve than normal non-ossified thyroid cartilage on VMIs equal to or higher than $95 \mathrm{keV}$ [50, 51]. Based on these studies, an improvement in accurate staging with DECT in the future seems feasible (Fig. 10).

\section{Bone Marrow Edema}

In general, bone marrow edema (BME) is best visualized by MR imaging techniques. With the DECT virtual noncalcium ( $\mathrm{VNCa}$ ) technique, it is possible to assess bone marrow edema with CT.

In the head and neck area, the presence of dental restorations, irregular tooth sockets, periapical and periodontal infection, other inflammatory reactions, and edema or sclerosis can give false positive results on both CT and MR imaging [52-54]. In daily practice, a combination of $\mathrm{CT}$ and MR imaging is often used to visualize subtle bone involvement in oncology, osteomyelitis, and osteonecrosis. 

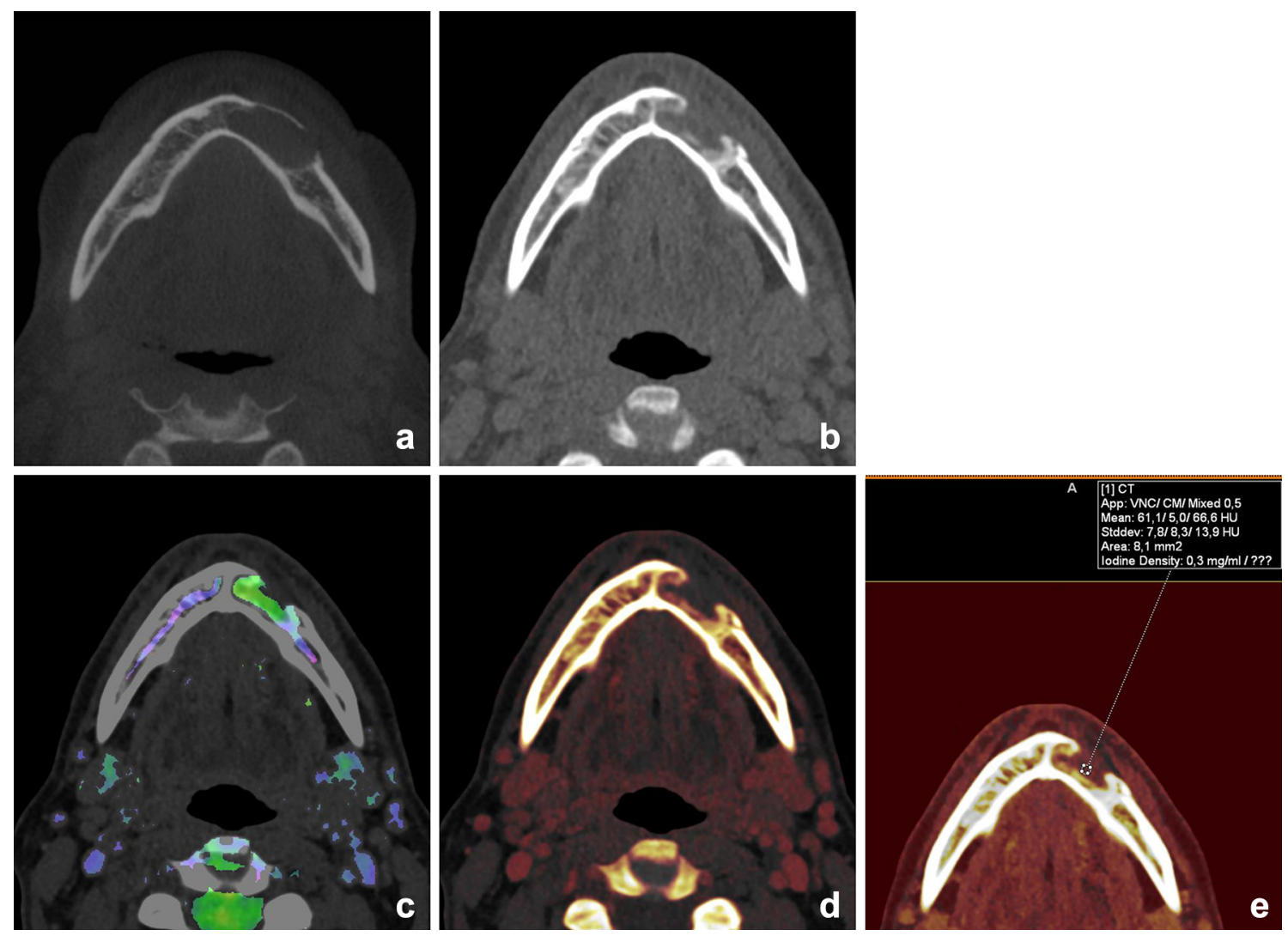

Fig. 11 A 50-year-old female underwent cystostomy for a left-sided keratocyst of the mandible (a). After 6 months, therapy was evaluated by $\operatorname{DECT}(\mathbf{b}$, mixed $)$. A residual lesion was found, with some residual edema (c, BME) and reparative bone apposition, but without signs of enhancement (d, e, iodine fusion (iodine concentration $0.3 \mathrm{mg} / \mathrm{ml}$ ))
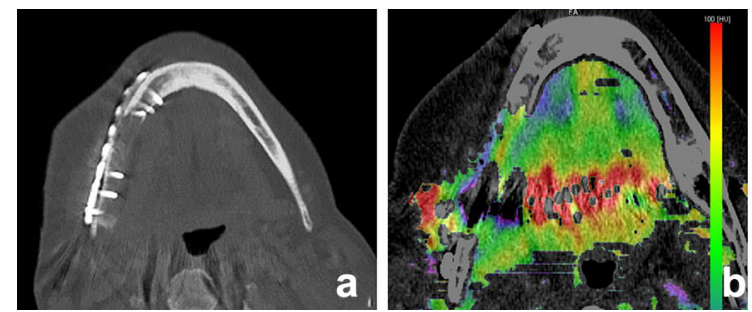

Fig. 12 A 60-year-old male presented with a persistent fistula after mandibular reconstruction, due to chronic osteomyelitis of the mandible. Depicted are $5 \mathrm{~mm}$ Maximum intensity projections of the mandible, showing metallic hardware next to an osteolytic mandible

No human studies in the head and neck area concerning DECT BME have been published.

Poort et al. investigated DECT as imaging technique for BME in osteoradionecrosis of the mandible in Göttingen mini-pigs. In this study, DECT was found to be an adequate single-modality imaging technique for simultaneous detection of structural bone changes, such as cortical disruptions, and BME. Further research is needed to investigate if DECT BME in the head and neck region is reliable [55]. Preliminary findings at our department demonstrate
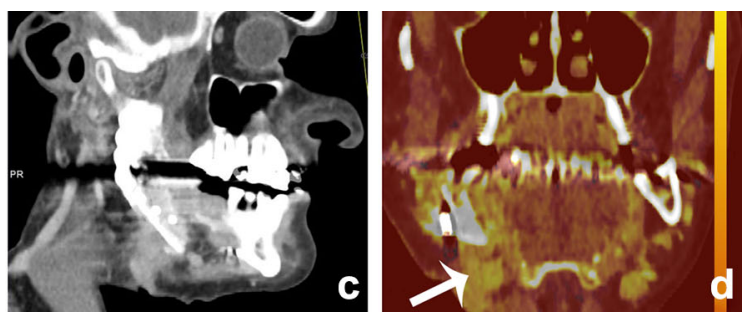

(a). BME images demonstrate bone marrow edema at the right mandible (b). The fistula is demonstrated at (c) and clearly enhanced at iodine fusion images at (d, arrow)

that it is feasible to show bone marrow edema in the human mandible (Figs. 9, 10, 11, 12 and 13).

\section{Cervical lymph Node Imaging}

The presence of metastatic lymph nodes in the neck significantly reduces the 5-year disease-specific survival rate in patients with HNSCC [56]. Since clinical physical examination of the neck has limitations, imaging is important, to confirm the NO status of the neck; to 

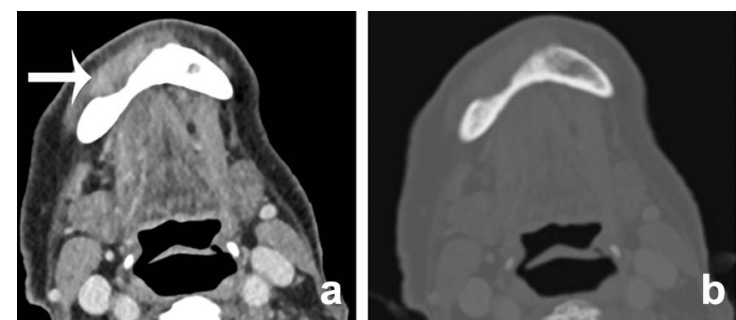

Fig. 13 A 90-year-old male was referred for staging of a gingival tumor originating at the right mandible. An MRI was contraindicated, and a DECT of the oral cavity was performed. Mixed images in soft tissue kernel (a) and bone kernel (b); iodine fusion images (c), and BME reconstructions (d). An enhancing tumor was found at the right
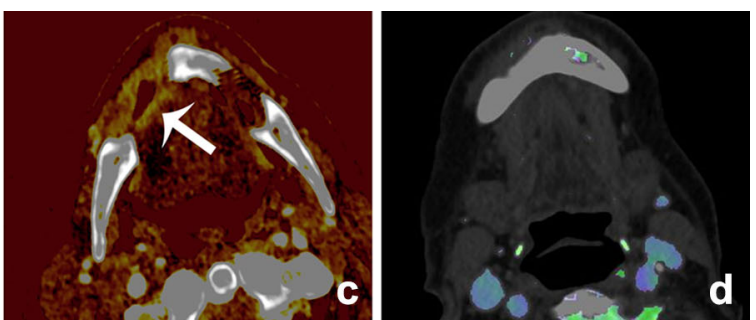

mandible (a, arrow) overlying an area of eroded or remodeled mandible (b) with sclerosis. Iodine fusion images additionally suggested invasion of the floor of the mouth, which was not seen on the mixed images (c, arrow). BME images did not show bone marrow edema. At histopathology, no osseous invasion was found
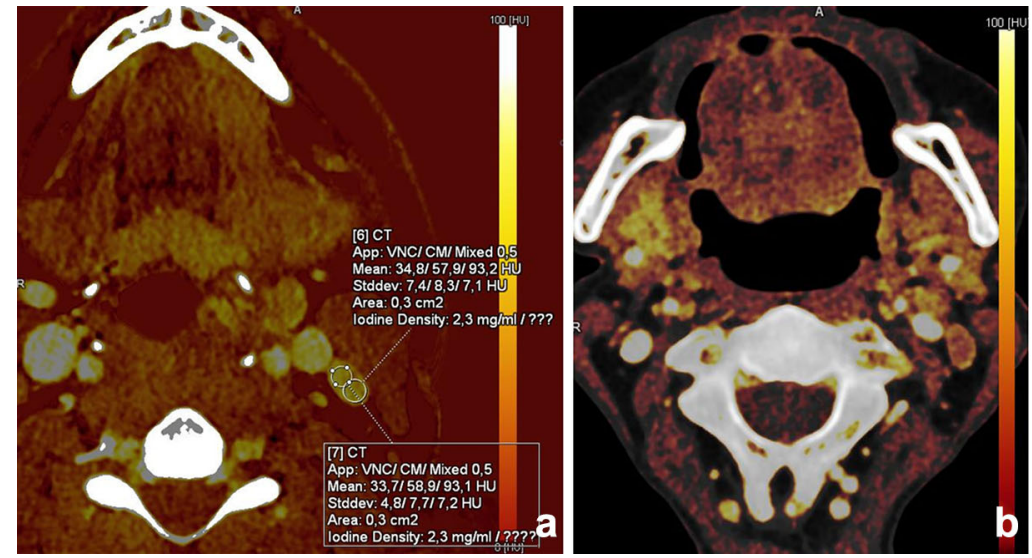

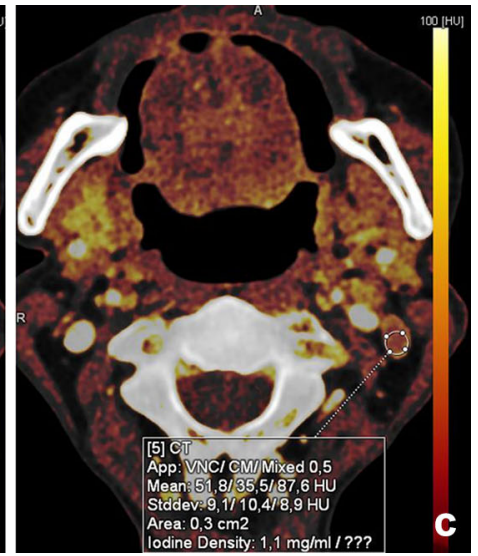

of a lymph node in a patient with oropharyngeal squamous cell carcinoma of the vallecula (iodine concentration $1.1 \mathrm{mg} / \mathrm{ml}$ ). Malignant lymph nodes have lower iodine uptake than normal or inflammatory lymph nodes, as is demonstrated in (b) and (c)
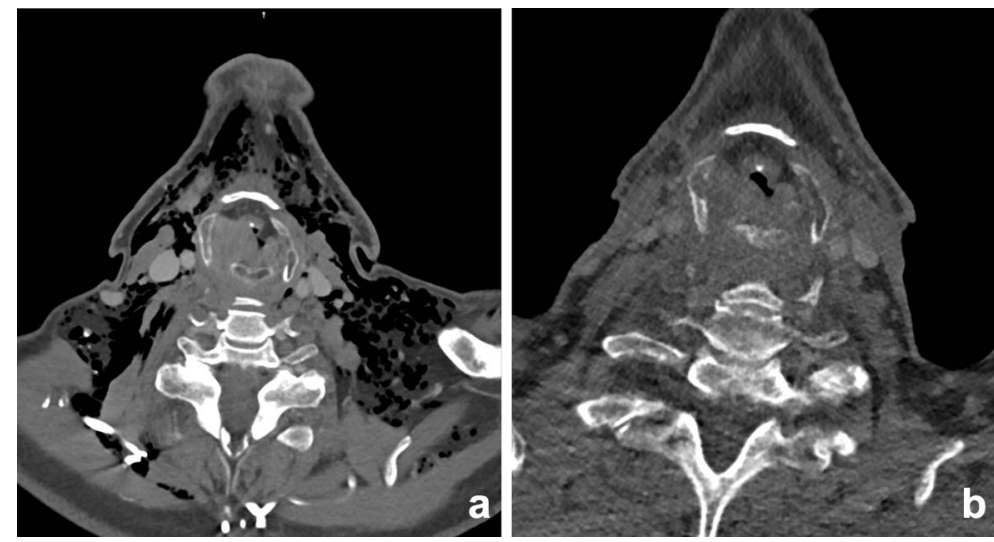

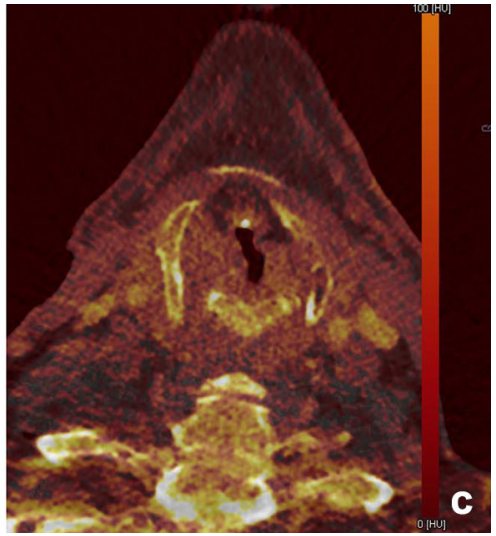

Fig. 15 An 81-year old male patient with a hypopharyngeal carcinoma from the piriform sinus, presented 6 months after chemoradiation to evaluate therapy response. The initial CT with the presence of a bilateral tumor of the piriform sinus and massive (postoperative) subcutaneous emphysema (a). A mixed DECT at one year (b) was

document lymphadenopathy contralateral to clinically palpable disease; and to assess the regional extent of disease, especially in relation to neurovascular structures and nodal surveillance for follow-up [57, 58]. made for evaluation of therapy effect and exclusion of residual tumor. Bilateral swelling is present, without clear enhancement at mixed imaging. At the iodine overlay images (c), no increased iodine uptake was present; benign posttreatment changes without residual or recurrent tumor were concluded

Several studies showed that DECT can be useful in lymph node imaging of the neck with good image quality and dose reduction [11, 23, 59-62]. Besides good image quality and lower image noise, functional and metabolic 

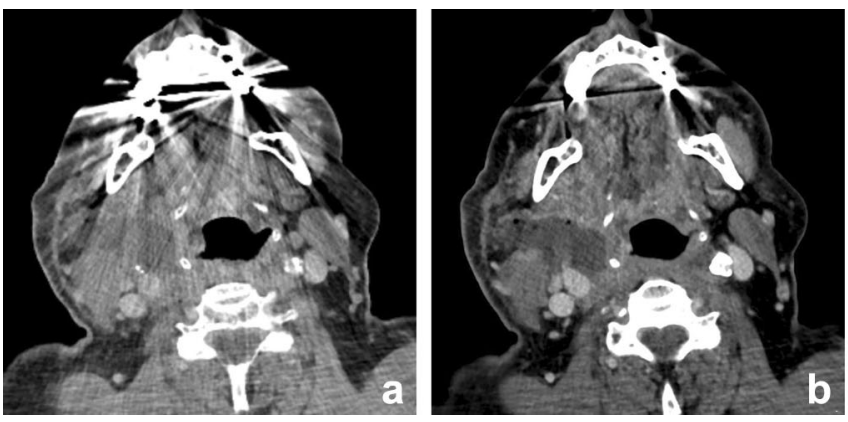

Fig. 16 Infection/abscesses: increased conspicuity at lower keV. A 65-year-old-male patient presented with a neck mass and a c-reactive protein of $180 \mu \mathrm{g} / \mathrm{ml}$ one week after carotid endarteriectomy. Mixed/ blended imaging showed right-sided swelling of the neck (a). Anterior of the sternocleidomastoid muscle, a fluid collection was visible, without obvious rim enhancement at mixed imaging. b-d The
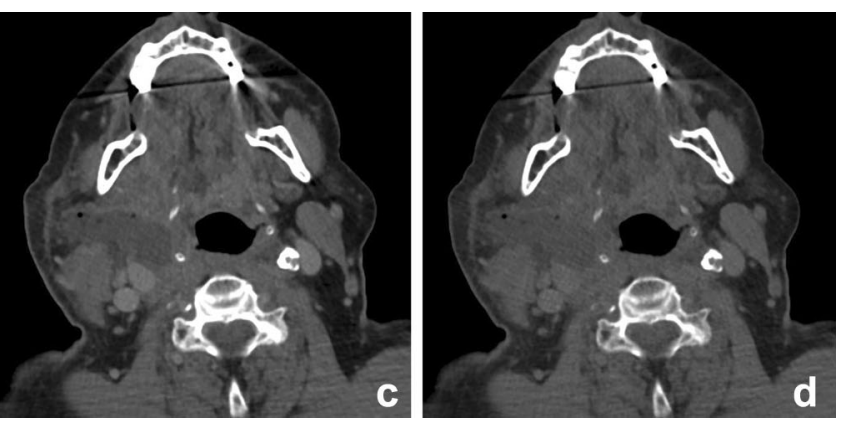

virtual mono-energetic reconstructions of 40 (b), 55 (c), and 70 (d) $\mathrm{keV}$. At higher keV, SNR was increased, but showed decrease of lesion conspicuity. At $40 \mathrm{keV}$, the lesion conspicuity and rim enhancement of the lesion were most optimal and suggestive of abscess formation. Small air bubbles were visible at the anterior part of the lesion
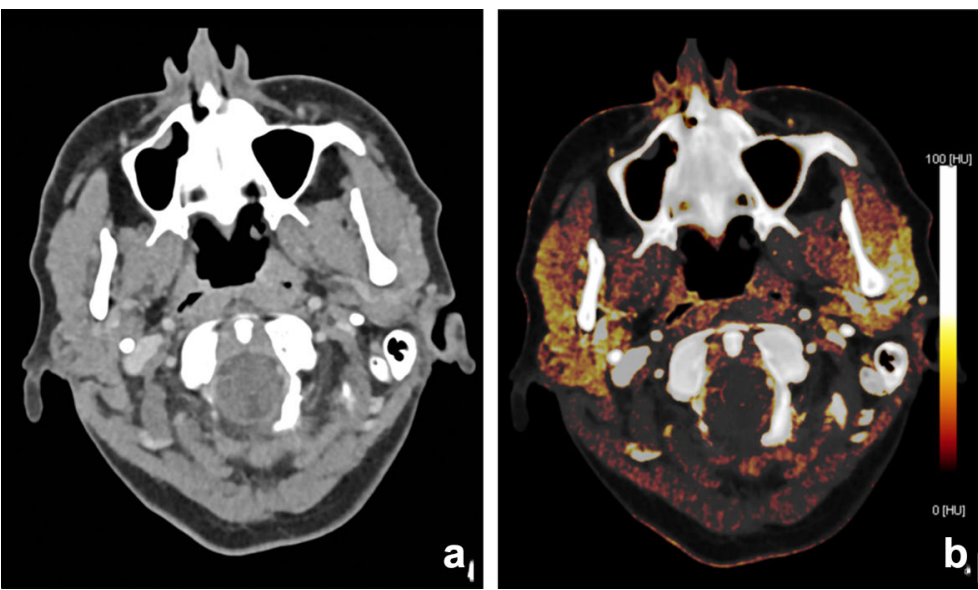

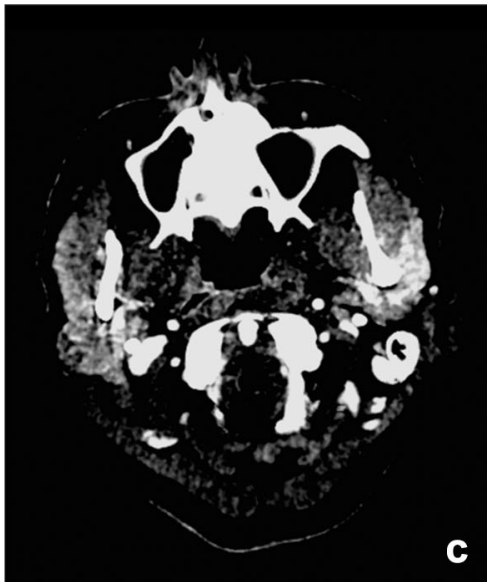

Fig. 17 A 48-year-old female with poor dental status presented with facial swelling and painful mouth opening. DECT was performed to rule out dental or neck abscesses, or arthritis of the temporomandibular joint. Mixed, 120-kVp-like images (a) demonstrated, besides a slight asymmetry no obvious abnormalities or fat stranding. The scan was initially interpreted as normal. However, Iodine map (b) and iodine fusion (c) images demonstrated clearly increased uptake of iodine at the superficial part of the parotid gland. Parotitis of the superficial lobe of the parotid gland was concluded

\section{Differentiating Recurrent Disease from Normal Posttreatment Changes}

Only a limited number of studies have been focusing on DECTs ability to differentiate between benign and malignant changes in patients with a history of neck malignancy. In a study of Srinivasan et al., the spectral HU curve was shown to be promising for differentiating benign posttreatment changes from malignant neck pathologies [66] (Fig. 15). More recently, Yamauchi et al. had similar results in their study for the spectral HU curves and corresponding results for the iodine concentration [67•]. In addition to the earlier study by Srinivasan, the latter authors compared the spectral HU curves at 40 and $70 \mathrm{keV}$ : the first theoretically showing the highest iodine concentration and the higher-energy-level curve tivity of $85 \%$ and a specificity of $87.5 \%[65 \cdot$. 

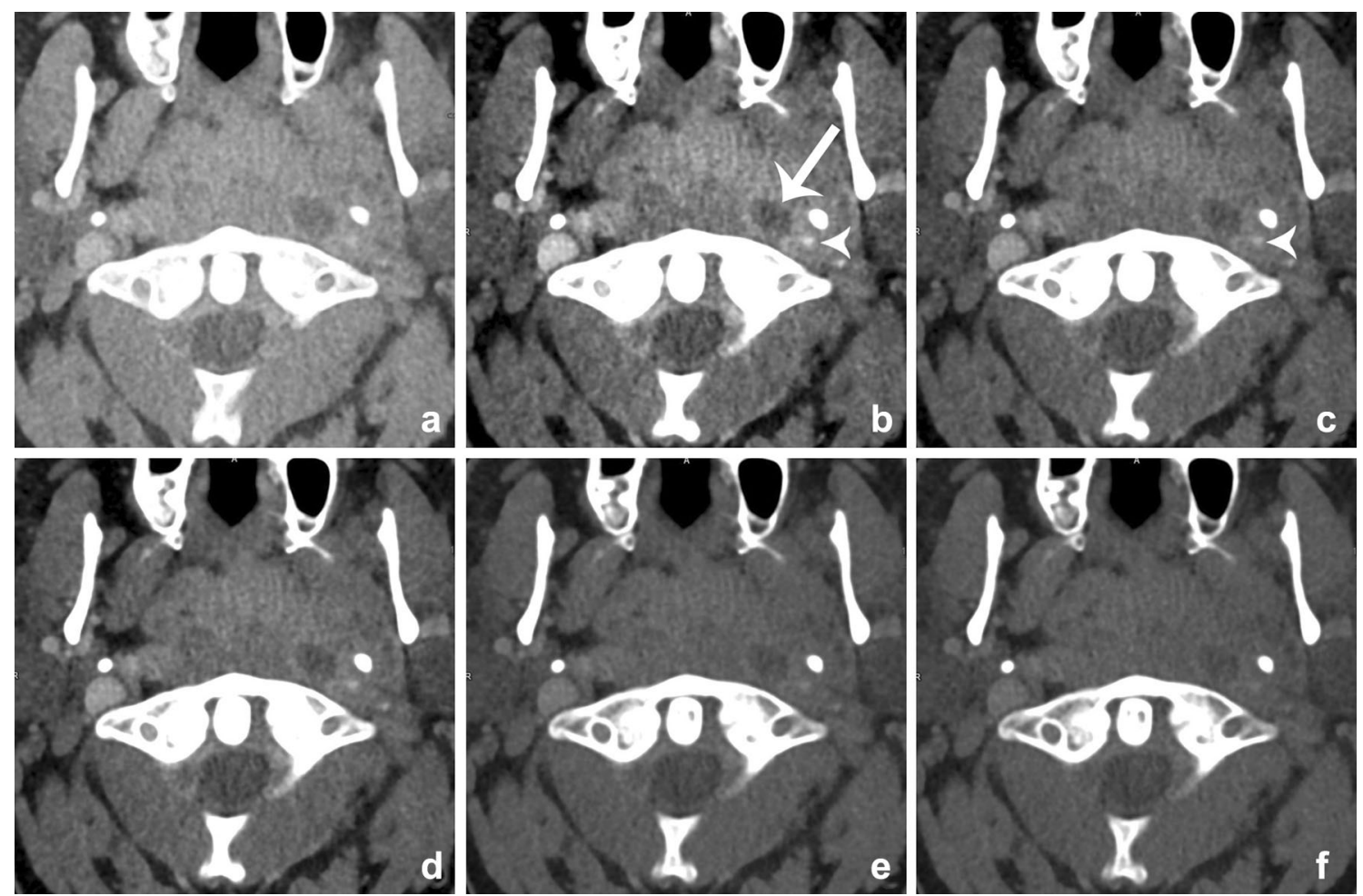

Fig. 18 Images of a young male patient with prevertebral abscesses. Blended images (50\%) (a) and VMIs (b-f). VMIs demonstrate improved visualization of the infiltration and abscesses at lower energies (arrow): $40 \mathrm{keV}$ (b), $50 \mathrm{keV}$ (c), $60 \mathrm{keV}$ (d), $70 \mathrm{keV}$ (e),

representing a standard MDCT with a $120-\mathrm{kVp}$ polychromatic X-ray beam. Comparison of the two showed better results for the curve at $40 \mathrm{keV}$.

\section{Infection and Inflammation}

Peritonsillar inflammation and abscess may present at any age, but they have the highest incidence rates in the adolescent population [68]. Therefore, especially in this patient group, scanning should be performed according to the ALARA principle. Scholtz et al. described the benefits of low-voltage images in peritonsillar abscess (PTA) compared with linearly blended $120-\mathrm{kVp}$ images [16]. First of all, they demonstrated an improved delineation of the PTA in $80-\mathrm{kVp}$ images compared with the $120-\mathrm{kVp}$ images. They reported a significant increase in SNR and rim-toabscess CNR (Fig. 16). Subjectively, the image sharpness was significantly better in the $80-\mathrm{kVp}$ images. Another possible advantage is the increased attenuation in adjacent vessels. This might improve operation planning in order to prevent bleeding in case of incision and drainage of the PTA. However, further studies must evaluate whether $80-\mathrm{kVp}$ scanning alone is sufficient in detection of PTA for additional dose reduction. Other possible advantages of DECT will still require the high-voltage scan. and $80 \mathrm{keV}$ (f). There is better visualization of the internal carotid artery at lower mono-energetic reconstructions, compared with blended image and higher-energy images, although at the cost of increased noise (arrowhead)

Wichmann et al. reported positive results in improved detection of sialoadenitis in three patients [60]. DECT of inflammatory diseases of the head and neck are thus far scarcely evaluated. Nevertheless, the results suggest a promising contribution of DECT due to the increased delineation, which can aid in an early detection and delineation of inflammation and abscesses (Figs. 17, 18).

\section{Parathyroid Tumors: Parathyroid Adenomas}

In patients with primary parahyperthyreoidism, adenomas are the most common cause. They are usually juxta thyroid single lesion, but multiple lesions and/or ectopic locations can occur. Treatment is surgical and has changed from bilateral exploratory to minimal invasive surgery. In order to be successful, preoperative identification and localization of the parathyroid adenomas is essential. A combination of sestamibi scanning and ultrasonography has proven effective with the addition of a third technique if results are not concordant [69].

Multiphase CT is accurate in localization of parathyroid adenomas due to different perfusion characteristics of thyroid gland, lymph nodes, and parathyroid adenomas. Due to awareness of radiation dose and diversity in protocol, the use was limited [70]. Gafton et al. showed that 

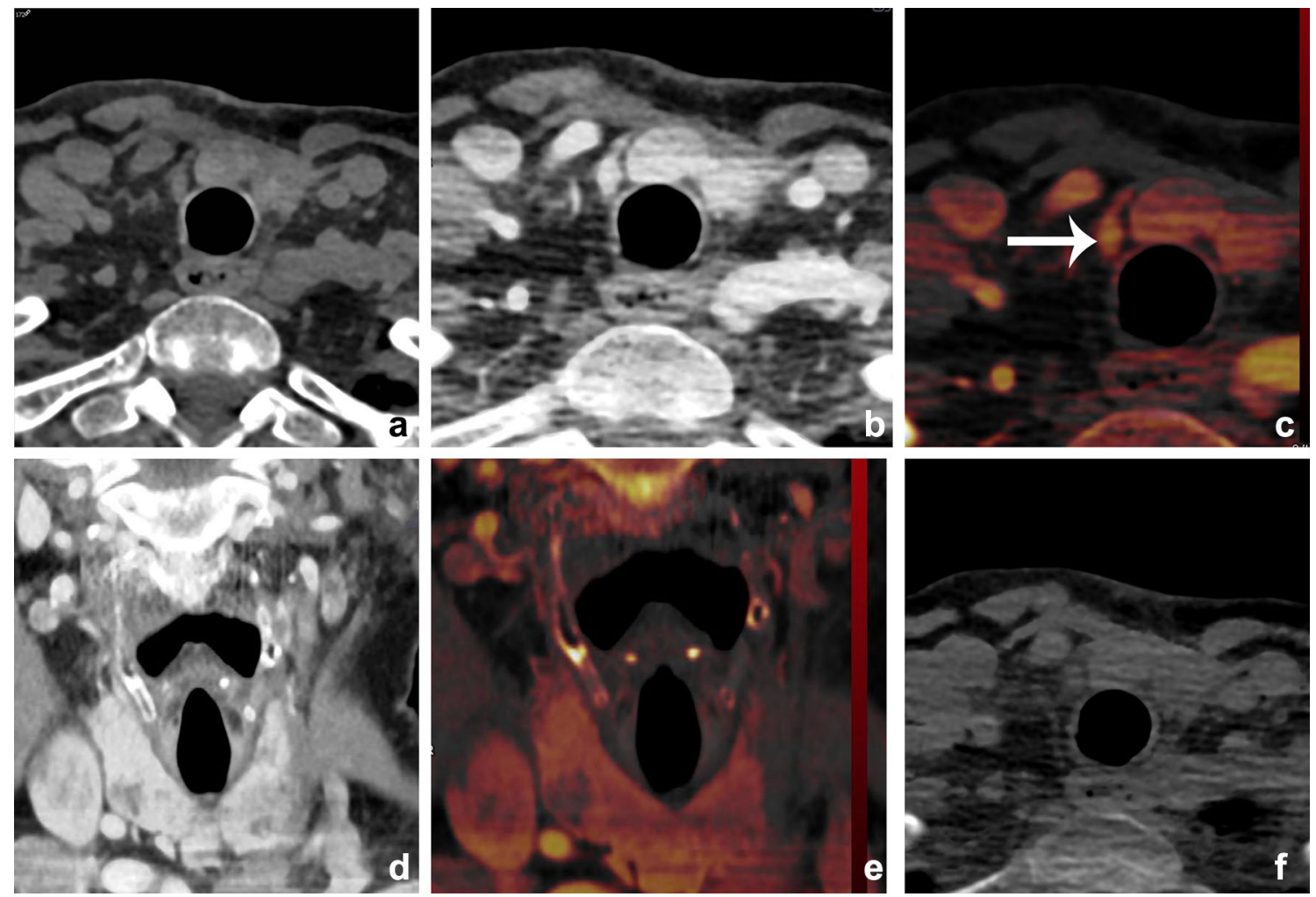

Fig. 19 A 63-year-old female presented with primary hyperparathyroidism. Initial Choline PET imaging and MIBI scanning were negative. A multiphase CT showed a small enhancing lesion just caudal to the right thyroid lobe, which was proven a parathyroid adenoma (arrow). Axial noncontrast images (a), 30-sec axial-mixed images (b), iodine fusion images (c), and coronal mixed (d), and fusion (e) images. A Virtual noncontrast image is shown at (f), the latter demonstrating more noise and streak artifacts than the true nonenhanced scan, although still diagnostic. Noncontrast CT: $80 \mathrm{kVp}$ 277 mAs; CTDI 5.34 mGy; DLP 113.4 mGycm. DECT $30 \mathrm{~s}$ : 80/150 kVP 61/31 mAs; CTDI 5.52 mGy; DLP 113.0 mGycm

\section{Thyroid Disease}

from other soft tissue structures by evaluating attenuation characteristics in arterial and venous phase. They could reduce radiation exposure by limiting the protocol to 2 phase imaging [71]. However, some believe this limitation in protocol reduces diagnostic confidence due to lack of a complete multiphase images. DECT is recommended to provide a more complete multiphase examination using VNC images and still reduce radiation exposure [72]. Forghani et al. demonstrated statistically significant differences in several DECT parameters partly depending on scan phase [73]. The arterial phase showed greater contrast between adenomas and lymph nodes, whereas the 55-s phase showed greater contrast between adenomas and thyroid gland. Although evidence is still limited, results indicate that multiphase DECT evaluation of parathyroid adenomas can enhance diagnostic accuracy. Moreover, the ability of DECT to calculate VNC images could theoretically lower radiation dose (Fig. 19).
Management of thyroid nodules remains challenging. DECT has been used to discriminate between benign and pathological nodules in a few studies by $\mathrm{Li}$ et al. [74-76]. These studies showed a difference in iodine uptake between benign and pathological nodules. Intralesional hemorrhage could be differentiated from solid nodules. Moreover, they could discriminate normal and metastatic lymph nodes from papillary thyroid carcinoma. It remains a matter of debate whether it is wise to use CE-CT in ruling out thyroid carcinoma.

\section{Radiotherapy Planning}

In radiotherapy, CT plays an important role for dose calculation in treatment planning, because of its relatively easy calibration of HU to electron densities [77, 78]. The possibility to calculate Zeff and electron density was 
already described by Hounsfield at the initial descriptions of DECT in 1973. It was demonstrated that a clinical DECT scanner was able to extract Zeff, and density $\rho$ of different tissue substitutes, next to $\Delta H U$ and $\rho e$ [79-81]. This suggested that when a large quantity of high-density and high atomic number structures are in the planning field, DECT-derived calculations show accurate and reliable inhomogeneity corrections in RT treatment planning [82].

There is an increasing interest in proton therapy because of its higher dose conformity and sparing of organs at risk compared with intensity-modulated radiation therapy [83]. Hudobivnik compared the proton therapy treatment planning of head tumors at the skull base to calculate the stopping powers while using SECT and DECT [84]. They confirmed a higher accuracy for DECT in their surrogate patients using a pencil beam algorithm. Zhu et al. confirmed in a phantom the dosimetric advantages in proton therapy treatment planning with DECT over the current approach based on SECT [85]. Whether this is clinically relevant needs to be investigated in future.

\section{Conclusion}

The use of DECT in head and neck imaging has been growing in the recent years. The advantages of additional DECT reconstructions at a comparable radiation dose are recognized by an increasing number of head and neck specialists. VMI and iodine characterization of DECT may play a major role in patients with HNSCC in detection and delineation of the tumor, resulting in more accurate staging. It can differentiate between malignant and benign lymph nodes based on iodine concentration, as well as between benign posttreatment changes and recurrent disease. VMI at higher $\mathrm{keV}$ is useful for reduction of metallic artifacts. Three material differentiation algorithms for identification of iodine and calcium can be used to assess cartilage and bone marrow infiltration, the latter being a new application in head and neck DECT. Imaging of infection and inflammation can be mitigated with DECT, and differential diagnosis can be facilitated with the use of spectral curves.

With the use of DECT, the inherent image information is more obvious due to the application of material characterization and differentiation, while maintaining a lower or equal radiation dose. This can especially be helpful in a difficult anatomical area like the head and neck.

\section{Compliance with Ethical Guidelines}

Conflict of interest Elise D. Roele, Veronique C. M. L. Timmer, Lauretta A. A. Vaassen, and Anna M. J. L. van Kroonenburgh, all declare no potential conflicts of interest. A. A. Postma reports speaker fees from Bayer and is a section editor for Current Radiology Reports.
Human and Animal Rights All reported studies/experiments with human or animal subjects performed by the authors have been previously published and complied with all applicable ethical standards (including the Helsinki declaration and its amendments, institutional/national research committee standards, and international/national/institutional guidelines).

Open Access This article is distributed under the terms of the Creative Commons Attribution 4.0 International License (http:// creativecommons.org/licenses/by/4.0/), which permits unrestricted use, distribution, and reproduction in any medium, provided you give appropriate credit to the original author(s) and the source, provide a link to the Creative Commons license, and indicate if changes were made.

\section{References}

Papers of particular interest, published recently, have been highlighted as:

- Of importance

•- Of major importance

1. Schulz B, Kuehling K, Kromen W, Siebenhandl P, Kerl MJ, Vogl $\mathrm{TJ}$, et al. Automatic bone removal technique in whole-body dualenergy CT angiography: performance and image quality. AJR Am J Roentgenol. 2012;199(5):W646-50. doi:10.2214/AJR.12. 9176.

2. Watanabe Y, Uotani K, Nakazawa T, Higashi M, Yamada N, Hori Y, et al. Dual-energy direct bone removal CT angiography for evaluation of intracranial aneurysm or stenosis: comparison with conventional digital subtraction angiography. Eur Radiol. 2009;19(4):1019-24. doi:10.1007/s00330-008-1213-5.

3. Graser A, Johnson TR, Hecht EM, Becker CR, Leidecker C, Staehler M, et al. Dual-energy CT in patients suspected of having renal masses: can virtual nonenhanced images replace true nonenhanced images? Radiology. 2009;252(2):433-40. doi:10. 1148/radiol.2522080557.

4. Boll DT, Patil NA, Paulson EK, Merkle EM, Simmons WN, Pierre SA, et al. Renal stone assessment with dual-energy multidetector CT and advanced postprocessing techniques: improved characterization of renal stone composition-pilot study. Radiology. 2009;250(3):813-20. doi:10.1148/radiol.2503080545.

5. Bauer RW, Kerl JM, Weber E, Weisser P, Korkusuz H, Lehnert $\mathrm{T}$, et al. Lung perfusion analysis with dual energy CT in patients with suspected pulmonary embolism-influence of window settings on the diagnosis of underlying pathologies of perfusion defects. Eur J Radiol. 2011;80(3):e476-82. doi:10.1016/j.ejrad. 2010.09.009.

6. Pontana F, Faivre JB, Remy-Jardin M, Flohr T, Schmidt B, Tacelli N, et al. Lung perfusion with dual-energy multidetectorrow CT (MDCT): feasibility for the evaluation of acute pulmonary embolism in 117 consecutive patients. Acad Radiol. 2008;15(12):1494-504. doi:10.1016/j.acra.2008.05.018.

7. Melzer R, Pauli C, Treumann T, Krauss B. Gout tophus detection-a comparison of dual-energy CT (DECT) and histology. Semin Arthritis Rheum. 2014;43(5):662-5. doi:10.1016/j.semarthrit. 2013.11.002.

8. Ray K. Imaging: dual-energy CT useful when assessing tophaceous gout. Nat Rev Rheumatol. 2012;8(2):64. doi:10.1038/ nrrheum.2011.220.

9. Postma AA, Hofman PA, Stadler AA, van Oostenbrugge RJ, Tijssen MP, Wildberger JE. Dual-energy CT of the brain and 
intracranial vessels. AJR Am J Roentgenol. 2012;199(5 Suppl): S26-33. doi:10.2214/AJR.12.9115.

10. Tijssen MP, Hofman PA, Stadler AA, van Zwam W, de Graaf R, van Oostenbrugge RJ, et al. The role of dual energy CT in differentiating between brain haemorrhage and contrast medium after mechanical revascularisation in acute ischaemic stroke. Eur Radiol. 2014;24(4):834-40. doi:10.1007/s00330-013-3073-x.

11. Vogl TJ, Schulz B, Bauer RW, Stover T, Sader R, Tawfik AM. Dual-energy CT applications in head and neck imaging. AJR Am J Roentgenol. 2012;199(5):S34-9. doi:10.2214/AJR.12.9113.

12. Johnson TR. Dual-energy CT: general principles. AJR Am J Roentgenol. 2012;199(5):S3-8. doi:10.2214/AJR.12.9116.

13. Marin D, Boll DT, Mileto A, Nelson RC. State of the art: dualenergy CT of the abdomen. Radiology. 2014;271(2):327-42. doi:10.1148/radiol.14131480.

14. Macari M, Spieler B, Kim D, Graser A, Megibow AJ, Babb J, et al. Dual-source dual-energy MDCT of pancreatic adenocarcinoma: initial observations with data generated at $80 \mathrm{kVp}$ and at simulated weighted-average $120 \mathrm{kVp}$. AJR Am J Roentgenol. 2010;194(1):W27-32. doi:10.2214/AJR.09.2737.

15. Holmes DR 3rd, Fletcher JG, Apel A, Huprich JE, Siddiki H, Hough DM, et al. Evaluation of non-linear blending in dualenergy computed tomography. Eur J Radiol. 2008;68(3):409-13. doi:10.1016/j.ejrad.2008.09.017.

16. Scholtz JE, Husers K, Kaup M, Albrecht MH, Beeres M, Bauer $\mathrm{RW}$, et al. Evaluation of image quality and dose reduction of 80 $\mathrm{kVp}$ neck computed tomography in patients with suspected peritonsillar abscess. Clin Radiol. 2015;70(8):e67-73. doi:10. 1016/j.crad.2015.04.009.

17. Albrecht MH, Trommer J, Wichmann JL, Scholtz JE, Martin SS, Lehnert T, et al. Comprehensive comparison of virtual monoenergetic and linearly blended reconstruction techniques in thirdgeneration dual-source dual-energy computed tomography angiography of the thorax and abdomen. Invest Radiol. 2016;51(9):582-90. doi:10.1097/RLI.0000000000000272.

18. Wichmann JL, Noske EM, Kraft J, Burck I, Wagenblast J, Eckardt A, et al. Virtual monoenergetic dual-energy computed tomography: optimization of kiloelectron volt settings in head and neck cancer. Invest Radiol. 2014;49(11):735-41. doi:10. 1097/RLI.0000000000000077.

19. $\mathrm{Yu} \mathrm{L}$, Leng $\mathrm{S}$, McCollough $\mathrm{CH}$. Dual-energy CT-based monochromatic imaging. AJR Am J Roentgenol. 2012;199(5 Suppl):S9-15. doi:10.2214/AJR.12.9121.

20. Albrecht MH, Scholtz JE, Husers K, Beeres M, Bucher AM, Kaup M, et al. Advanced image-based virtual monoenergetic dual-energy CT angiography of the abdomen: optimization of kiloelectron volt settings to improve image contrast. Eur Radiol. 2016;26(6):1863-70. doi:10.1007/s00330-015-3970-2.

21. Albrecht MH, Scholtz JE, Kraft J, Bauer RW, Kaup M, Dewes P, et al. Assessment of an advanced monoenergetic reconstruction technique in dual-energy computed tomography of head and neck cancer. Eur Radiol. 2015;25(8):2493-501. doi:10.1007/s00330015-3627-1.

22. Ho LM, Yoshizumi TT, Hurwitz LM, Nelson RC, Marin D, Toncheva $G$, et al. Dual energy versus single energy MDCT: measurement of radiation dose using adult abdominal imaging protocols. Acad Radiol. 2009;16(11):1400-7. doi:10.1016/j.acra. 2009.05.002.

23. Tawfik AM, Kerl JM, Razek AA, Bauer RW, Nour-Eldin NE, Vogl TJ, et al. Image quality and radiation dose of dual-energy $\mathrm{CT}$ of the head and neck compared with a standard $120-\mathrm{kVp}$ acquisition. AJNR Am J Neuroradiol. 2011;32(11):1994-9. doi:10.3174/ajnr.A2654.

24. Zhu X, McCullough WP, Mecca P, Servaes S, Darge K. Dualenergy compared to single-energy $\mathrm{CT}$ in pediatric imaging: a phantom study for DECT clinical guidance. Pediatr Radiol. 2016;. doi:10.1007/s00247-016-3668-x.

25. Barrett JF, Keat N. Artifacts in CT: recognition and avoidance. Radiographics. 2004;24(6):1679-91. doi:10.1148/rg.246045065.

26. Kuchenbecker S, Faby S, Sawall S, Lell M, Kachelriess M. Dual energy CT: how well can pseudo-monochromatic imaging reduce metal artifacts? Med Phys. 2015;42(2):1023-36. doi:10.1118/1. 4905106.

27. Tanaka R, Hayashi T, Ike M, Noto Y, Goto TK. Reduction of dark-band-like metal artifacts caused by dental implant bodies using hypothetical monoenergetic imaging after dual-energy computed tomography. Oral Surg Oral Med Oral Pathol Oral Radiol. 2013;115(6):833-8. doi:10.1016/j.0ooo.2013.03.014.

28. Takrouri HS, Alnassar MM, Amirabadi A, Babyn PS, Moineddin $\mathrm{R}$, Padfield NL, et al. Metal artifact reduction: added value of rapid-kilovoltage-switching dual-energy $\mathrm{CT}$ in relation to singleenergy CT in a piglet animal model. AJR Am J Roentgenol. 2015;205(3):W352-9. doi:10.2214/AJR.14.12547.

29. Wang Y, Qian B, Li B, Qin G, Zhou Z, Qiu Y, et al. Metal artifacts reduction using monochromatic images from spectral CT: evaluation of pedicle screws in patients with scoliosis. Eur J Radiol. 2013;82(8):e360-6. doi:10.1016/j.ejrad.2013.02.024.

30. Zhou C, Zhao YE, Luo S, Shi H, Li L, Zheng L, et al. Monoenergetic imaging of dual-energy CT reduces artifacts from implanted metal orthopedic devices in patients with factures. Acad Radiol. 2011;18(10):1252-7. doi:10.1016/j.acra.2011.05. 009.

31. Bamberg F, Dierks A, Nikolaou K, Reiser MF, Becker CR, Johnson TR. Metal artifact reduction by dual energy computed tomography using monoenergetic extrapolation. Eur Radiol. 2011;21(7):1424-9. doi:10.1007/s00330-011-2062-1.

32. Guggenberger R, Winklhofer S, Osterhoff G, Wanner GA, Fortunati $\mathrm{M}$, Andreisek $\mathrm{G}$, et al. Metallic artefact reduction with monoenergetic dual-energy CT: systematic ex vivo evaluation of posterior spinal fusion implants from various vendors and different spine levels. Eur Radiol. 2012;22(11):2357-64. doi:10. 1007/s00330-012-2501-7.

33. Srinivasan A, Hoeffner E, Ibrahim M, Shah GV, LaMarca F, Mukherji SK. Utility of dual-energy CT virtual keV monochromatic series for the assessment of spinal transpedicular hardwarebone interface. AJR Am J Roentgenol. 2013;201(4):878-83. doi:10.2214/AJR.12.9736.

34. Stolzmann P, Winklhofer S, Schwendener N, Alkadhi H, Thali MJ, Ruder TD. Monoenergetic computed tomography reconstructions reduce beam hardening artifacts from dental restorations. Forensic Sci Med Pathol. 2013;9(3):327-32. doi:10.1007/ s12024-013-9420-z.

35. De Crop A, Casselman J, Van Hoof T, Dierens M, Vereecke E, Bossu N, et al. Analysis of metal artifact reduction tools for dental hardware in CT scans of the oral cavity: $\mathrm{kVp}$, iterative reconstruction, dual-energy $\mathrm{CT}$, metal artifact reduction software: does it make a difference? Neuroradiology. 2015;57(8):841-9. doi:10.1007/s00234-015-1537-1.

36. Bongers MN, Schabel C, Thomas C, Raupach R, Notohamiprodjo $\mathrm{M}$, Nikolaou $\mathrm{K}$, et al. Comparison and combination of dual-energy- and iterative-based metal artefact reduction on hip prosthesis and dental implants. PLoS ONE. 2015;10(11):e0143584. doi:10.1371/journal.pone.0143584.

37. Lee MJ, Kim S, Lee SA, Song HT, Huh YM, Kim DH, et al. Overcoming artifacts from metallic orthopedic implants at highfield-strength MR imaging and multi-detector CT. Radiographics. 2007;27(3):791-803. doi:10.1148/rg.273065087.

38. Shah JP, Gil Z. Current concepts in management of oral cancer-surgery. Oral Oncol. 2009;45(4-5):394-401. doi:10.1016/j.oraloncology. 2008.05.017. 
39. Li C, Yang W, Men Y, Wu F, Pan J, Li L. Magnetic resonance imaging for diagnosis of mandibular involvement from head and neck cancers: a systematic review and meta-analysis. PLoS ONE. 2014;9(11):e112267. doi:10.1371/journal.pone.0112267.

40. Li C, Men Y, Yang W, Pan J, Sun J, Li L. Computed tomography for the diagnosis of mandibular invasion caused by head and neck cancer: a systematic review comparing contrast-enhanced and plain computed tomography. J Oral Maxillofac Surg. 2014; 72(8):1601-15. doi:10.1016/j.joms.2014.02.014.

41. Tawfik AM, Kerl JM, Bauer RW, Nour-Eldin NE, Naguib NN, Vogl TJ, et al. Dual-energy CT of head and neck cancer: average weighting of low- and high-voltage acquisitions to improve lesion delineation and image quality-initial clinical experience. Invest Radiol. 2012;47(5):306-11. doi:10.1097/RLI.0b013e31821e30 62 .

42. Scholtz JE, Husers K, Kaup M, Albrecht M, Schulz B, Frellesen $\mathrm{C}$, et al. Non-linear image blending improves visualization of head and neck primary squamous cell carcinoma compared to linear blending in dual-energy CT. Clin Radiol. 2015;70(2): 168-75. doi:10.1016/j.crad.2014.10.018.

43. Scholtz JE, Kaup M, Kraft J, Noske EM, Scheerer F, Schulz B, et al. Objective and subjective image quality of primary and recurrent squamous cell carcinoma on head and neck low-tubevoltage $80-\mathrm{kVp}$ computed tomography. Neuroradiology. 2015;57(6):645-51. doi:10.1007/s00234-015-1512-x.

44. Toepker M, Czerny C, Ringl H, Fruehwald-Pallamar J, Wolf F, Weber M, et al. Can dual-energy CT improve the assessment of tumor margins in oral cancer? Oral Oncol. 2014;50(3):221-7. doi:10.1016/j.oraloncology.2013.12.001.

45. • Lam S, Gupta R, Levental M, Yu E, Curtin HD, Forghani R. Optimal virtual monochromatic images for evaluation of normal tissues and head and neck cancer using dual-energy CT. AJNR Am J Neuroradiol. 2015;36(8):1518-24. doi:10.3174/ajnr.A4314. Defined the optimal mono-energetic energy levels. They concluded that a multi-parametric approach is desirable, a maximum delineation of the tumor is at low monoenergetic energy (40 keV), while optimal SNR is at higher energy $(65 \mathrm{keV})$.

46. NCCN Clinical Practice Guidelines in Oncology, Head and Neck Cancers [database on the Internet]2016. Available from: https:// www.nccn.org/professionals/physician_gls/pdf/head-and-neck. pdf. Accessed: 27 Oct 2016.

47. Becker M, Zbären P, Delavelle J, Kurt AM, Egger C, Rüfenacht DA, et al. Neoplastic invasion of the laryngeal cartilage: reassessment of criteria for diagnosis at CT. Radiology. 1997;203(2):521-32. doi:10.1148/radiology.203.2.9114116.

48. Becker M, Zbären P, Casselman JW, Kohler R, Dulguerov P, Becker CD. Neoplastic invasion of laryngeal cartilage: reassessment of criteria for diagnosis at MR imaging. Radiology. 2008;249(2):551-9. doi:10.1148/radiol.2492072183.

49. Kuno H, Onaya H, Iwata R, Kobayashi T, Fujii S, Hayashi R, et al. Evaluation of cartilage invasion by laryngeal and hypopharyngeal squamous cell carcinoma with dual-energy CT. Radiology. 2012;265(2):488-96. doi:10.1148/radiol.12111719.

50. Forghani R, Levental M, Gupta R, Lam S, Dadfar N, Curtin HD. Different spectral hounsfield unit curve and high-energy virtual monochromatic image characteristics of squamous cell carcinoma compared with nonossified thyroid cartilage. AJNR Am J Neuroradiol. 2015;36(6):1194-200. doi:10.3174/ajnr.A4253.

51. Forghani R. Advanced dual-energy CT for head and neck cancer imaging. Expert Rev Anticancer Ther. 2015;15(12):1489-501. doi:10.1586/14737140.2015.1108193.

52. Van Cann EM, Koole R, Oyen WJ, de Rooy JW, de Wilde PC, Slootweg PJ, et al. Assessment of mandibular invasion of squamous cell carcinoma by various modes of imaging: constructing a diagnostic algorithm. Int J Oral Maxillofac Surg. 2008;37(6): 535-41. doi:10.1016/j.ijom.2008.02.009.
53. Van Cann EM, Rijpkema M, Heerschap A, van der Bilt A, Koole $\mathrm{R}$, Stoelinga PJ. Quantitative dynamic contrast-enhanced MRI for the assessment of mandibular invasion by squamous cell carcinoma. Oral Oncol. 2008;44(12):1147-54. doi:10.1016/j. oraloncology.2008.02.009.

54. Deshpande SS, Donneys A, Farberg AS, Tchanque-Fossuo CN, Felice PA, Buchman SR. Quantification and characterization of radiation-induced changes to mandibular vascularity using microcomputed tomography. Ann Plast Surg. 2014;72(1):100-3. doi:10.1097/SAP.0b013e318255a57d.

55. Poort LJ SA, Ludlage JHB, Hoebers FJP, Kessler PAWH, Postma AA. Detection of bone marrow edema pattern with Dual-Energy $\mathrm{CT}$ of the pig mandible treated with radiotherapy and surgery compared with MR. JCAT. 2017; [in press].

56. Layland MK, Sessions DG, Lenox J. The influence of lymph node metastasis in the treatment of squamous cell carcinoma of the oral cavity, oropharynx, larynx, and hypopharynx: N0 versus $\mathrm{N}+$. Laryngoscope. 2005;115(4):629-39. doi:10.1097/01.mlg. 0000161338.54515.b1.

57. Chong V. Cervical lymphadenopathy: what radiologists need to know. Cancer Imaging. 2004;4(2):116-20. doi:10.1102/14707330.2004.0020.

58. Liang H, Li A, Li Y, Cheng H, Zhao Q, Li J, et al. A retrospective study of dual-energy CT for clinical detecting of metastatic cervical lymph nodes in laryngeal and hypopharyngeal squamous cell carcinoma. Acta Otolaryngol. 2015;135(7):722-8. doi:10. 3109/00016489.2015.1015164.

59. Paul J, Mbalisike EC, Nour-Eldin NE, Vogl TJ. Dual-source 128-slice MDCT neck: radiation dose and image quality estimation of three different protocols. Eur $\mathrm{J}$ Radiol. 2013;82(5):787-96. doi:10.1016/j.ejrad.2012.11.024.

60. Wichmann JL, Kraft J, Noske EM, Bodelle B, Burck I, Scholtz $\mathrm{JE}$, et al. Low-tube-voltage $80-\mathrm{kVp}$ neck CT: evaluation of diagnostic accuracy and interobserver agreement. AJNR Am J Neuroradiol. 2014;35(12):2376-81. doi:10.3174/ajnr.A4052.

61. Gnannt R, Winklehner A, Goetti R, Schmidt B, Kollias S, Alkadhi H. Low kilovoltage CT of the neck with $70 \mathrm{kVp}$ : comparison with a standard protocol. AJNR Am J Neuroradiol. 2012;33(6):1014-9. doi:10.3174/ajnr.A2910.

62. Hoang JK, Vanka J, Ludwig BJ, Glastonbury CM. Evaluation of cervical lymph nodes in head and neck cancer with CT and MRI: tips, traps, and a systematic approach. AJR Am J Roentgenol. 2013;200(1):W17-25. doi:10.2214/AJR.12.8960.

63. Schmid-Bindert G, Henzler T, Chu TQ, Meyer M, Nance JW Jr, Schoepf UJ, et al. Functional imaging of lung cancer using dual energy CT: how does iodine related attenuation correlate with standardized uptake value of 18FDG-PET-CT? Eur Radiol. 2012;22(1):93-103. doi:10.1007/s00330-011-2230-3.

64. Sadick M, Schoenberg SO, Hoermann K, Sadick H. Current oncologic concepts and emerging techniques for imaging of head and neck squamous cell cancer. Laryngorhinootologie. 2012;91(1):S27-47. doi:10.1055/s-0031-1297259.

65. - Tawfik AM, Razek AA, Kerl JM, Nour-Eldin NE, Bauer R, Vogl TJ. Comparison of dual-energy CT-derived iodine content and iodine overlay of normal, inflammatory and metastatic squamous cell carcinoma cervical lymph nodes. Eur Radiol. 2014;24(3):574-80. doi:10.1007/s00330-013-3035-3. Differentiated malignant from reactive and benign lymph nodes by measuring the iodine concentration. They could reach a sensitivity of $85 \%$ to detect malignant lymph nodes by using a cut-of value of $285 \mathrm{mg} / \mathrm{ml}$ iodine.

66. Srinivasan A, Parker RA, Manjunathan A, Ibrahim M, Shah GV, Mukherji SK. Differentiation of benign and malignant neck pathologies: preliminary experience using spectral computed tomography. J Comput Assist Tomogr. 2013;37(5):666-72. doi:10.1097/RCT.0b013e3182976365. 
67. - Yamauchi H, Buehler M, Goodsitt MM, Keshavarzi N, Srinivasan A. Dual-energy CT-based differentiation of benign posttreatment changes from primary or recurrent malignancy of the head and neck: comparison of spectral Hounsfield units at 40 and $70 \mathrm{keV}$ and iodine concentration. AJR Am J Roentgenol. 2016;206(3):580-7. doi:10.2214/AJR.15.14896. Demonstrated that DECT-derived spectral Hounsfield units at $40 \mathrm{keV}$ and iodine concentration may be superior to spectral Hounsfield units at $70 \mathrm{keV}$ in differentiating benign posttreatment changes from primary or recurrent head and neck malignancies.

68. Risberg S, Engfeldt P, Hugosson S. Incidence of peritonsillar abscess and relationship to age and gender: retrospective study. Scand J Infect Dis. 2008;40(10):792-6. doi:10.1080/003655408 02195226.

69. Munk RS, Payne RJ, Luria BJ, Hier MP, Black MJ. Preoperative localization in primary hyperparathyroidism. J Otolaryngol Head Neck Surg. 2008;37(3):347-54.

70. Hunter GJ, Schellingerhout D, Vu TH, Perrier ND, Hamberg LM. Accuracy of four-dimensional CT for the localization of abnormal parathyroid glands in patients with primary hyperparathyroidism. Radiology. 2012;264(3):789-95. doi:10.1148/radiol. 12110852.

71. Gafton AR, Glastonbury CM, Eastwood JD, Hoang JK. Parathyroid lesions: characterization with dual-phase arterial and venous enhanced CT of the neck. AJNR Am J Neuroradiol. 2012;33(5):949-52. doi:10.3174/ajnr.A2885.

72. Lau D, Yang H, Kei PL. Dual-energy 4-phase CT scan in primary hyperparathyroidism. AJNR Am J Neuroradiol. 2013;34(8): E91-3. doi:10.3174/ajnr.A3657.

73. Forghani R, Roskies M, Liu X, Tan X, Mlynarek A, Payne RJ, et al. Dual-energy CT characteristics of parathyroid adenomas on 25-and 55-second 4D-CT acquisitions: preliminary experience. J Comput Assist Tomogr. 2016;40(5):806-14. doi:10.1097/RCT. 0000000000000442.

74. Li M, Zheng X, Gao F, Xiao L, Hua Y. Spectral CT imaging of intranodular hemorrhage in cases with challenging benign thyroid nodules. Radiol Med. (Torino). 2016;121(4):279-90. doi:10. 1007/s11547-015-0601-6.

75. Li M, Zheng X, Li J, Yang Y, Lu C, Xu H, et al. Dual-energy computed tomography imaging of thyroid nodule specimens: comparison with pathologic findings. Invest Radiol. 2012;47(1): 58-64. doi:10.1097/RLI.0b013e318229fef3.
76. Liu X, Ouyang D, Li H, Zhang R, Lv Y, Yang A, et al. Papillary thyroid cancer: dual-energy spectral CT quantitative parameters for preoperative diagnosis of metastasis to the cervical lymph nodes. Radiology. 2015;275(1):167-76. doi:10.1148/radiol.1414 0481.

77. van Elmpt W, Landry G, Das M, Verhaegen F. Dual energy CT in radiotherapy: current applications and future outlook. Radiother Oncol. 2016;119(1):137-44. doi:10.1016/j.radonc.2016.02.026.

78. Saito M. Potential of dual-energy subtraction for converting CT numbers to electron density based on a single linear relationship. Med Phys. 2012;39(4):2021-30. doi:10.1118/1.3694111.

79. Saito M. Optimized low-kV spectrum of dual-energy CT equipped with high-kV tin filtration for electron density measurements. Med Phys. 2011;38(6):2850-8. doi:10.1118/1.3584200.

80. Saito M. Technical Note: relation between dual-energy subtraction of CT images for electron density calibration and virtual monochromatic imaging. Med Phys. 2015;42(7):4088-93. doi:10. 1118/1.4921999.

81. Landry G, Reniers B, Granton PV, van Rooijen B, Beaulieu L, Wildberger JE, et al. Extracting atomic numbers and electron densities from a dual source dual energy CT scanner: experiments and a simulation model. Radiother Oncol. 2011;100(3):375-9. doi:10.1016/j.radonc.2011.08.029.

82. Tsukihara M, Noto Y, Hayakawa T, Saito M. Conversion of the energy-subtracted CT number to electron density based on a single linear relationship: an experimental verification using a clinical dual-source CT scanner. Phys Med Biol. 2013;58(9): N135-44. doi:10.1088/0031-9155/58/9/N135.

83. van de Water S, Kooy HM, Heijmen BJ, Shortening delivery times of intensity modulated proton therapy by reducing proton energy layers during treatment plan optimization. Int $\mathrm{J}$ Radiat Oncol Biol Phys. 2015;92(2):460-8. doi:10.1016/j.ijrobp.2015. 01.031 .

84. Hudobivnik N, Schwarz F, Johnson T, Agolli L, Dedes G, Tessonnier $\mathrm{T}$, et al. Comparison of proton therapy treatment planning for head tumors with a pencil beam algorithm on dual and single energy CT images. Med Phys. 2016;43(1):495. doi:10.1118/1. 4939106.

85. Zhu J, Penfold SN. Dosimetric comparison of stopping power calibration with dual-energy $\mathrm{CT}$ and single-energy $\mathrm{CT}$ in proton therapy treatment planning. Med Phys. 2016;43(6):2845. doi:10. 1118/1.4948683. 